\title{
Near-Quadratic Lower Bounds for Two-Pass Graph Streaming Algorithms
}

\author{
Sepehr Assadi* $\quad$ Ran Raz ${ }^{\dagger}$
}

\begin{abstract}
We prove that any two-pass graph streaming algorithm for the $s$ - $t$ reachability problem in $n$-vertex directed graphs requires near-quadratic space of $n^{2-o(1)}$ bits. As a corollary, we also obtain near-quadratic space lower bounds for several other fundamental problems including maximum bipartite matching and (approximate) shortest path in undirected graphs.

Our results collectively imply that a wide range of graph problems admit essentially no non-trivial streaming algorithm even when two passes over the input is allowed. Prior to our work, such impossibility results were only known for single-pass streaming algorithms, and the best two-pass lower bounds only ruled out $o\left(n^{7 / 6}\right)$ space algorithms, leaving open a large gap between (trivial) upper bounds and lower bounds.
\end{abstract}

\footnotetext{
*(sepehr.assadi@rutgers.edu) Department of Computer Science, Rutgers University. Part of this work was done while the author was a postdoctoral researcher at Princeton University and was supported in part by the Simons Collaboration on Algorithms and Geometry.

${ }^{\dagger}$ (ran.raz.mail@gmail.com) Department of Computer Science, Princeton University. Research supported by the Simons Collaboration on Algorithms and Geometry, by a Simons Investigator Award, and by the National Science Foundation grants No. CCF-171477 and CCF-2007462.
} 


\section{Contents}

1 Introduction $\quad 1$

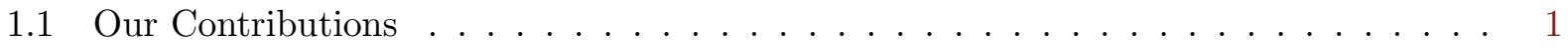

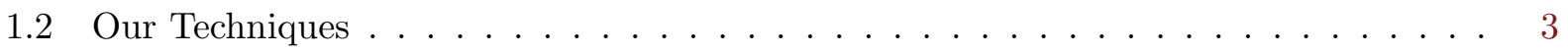

1.3 Subsequent Work . . . . . . . . . . . . . . . . . . . . 4

2 Preliminaries $\quad 4$

2.1 Communication Complexity and Information Complexity . . . . . . . . . . . 5

2.2 Ruzsa-Szemerédi Graphs . . . . . . . . . . . . . . . . . . . . . . . . 5

3 A New Lower Bound for the Set Intersection Problem 5

3.1 The Lower Bound for $\varepsilon$-Internal Solving of Set Intersection $\ldots \ldots$. . . . . . . . . 7

4 The Unique-Reach Communication Problem 11

4.1 Distribution $\mathcal{D}_{\mathrm{UR}}$ in Theorem $2 \ldots \ldots \ldots \ldots \ldots \ldots$

4.2 Proof of Theorem $2 \ldots \ldots \ldots \ldots \ldots$

4.3 The Inverse Unique-Reach Problem . . . . . . . . . . . . . . . . . . . . 15

5 The st-Reachability Communication Problem 16

5.1 A Hard Distribution for st-reachability . . . . . . . . . . . . . . . . . 16

5.2 Setup and Notation . . . . . . . . . . . . . . . . . . . . 17

5.3 Part One: The First Round of Communication . . . . . . . . . . . . . . . . . 19

5.4 Part Two: The Second Round of Communication . . . . . . . . . . . . . . . . . 21

5.5 Concluding the Proof of Theorem $3 \ldots \ldots \ldots \ldots$

6 Graph Streaming Lower Bounds $\quad 24$

6.1 Directed Reachability . . . . . . . . . . . . . . . . . . . 24

6.2 Bipartite Perfect Matching . . . . . . . . . . . . . . . . 25

6.3 Single-Source Shortest Path . . . . . . . . . . . . . . . . . 25

A Basic Information Theory Facts 33 


\section{Introduction}

Graph streaming algorithms process the input graph with $n$ known vertices by making one or a few passes over the sequence of its unknown edges (given in an arbitrary order) and using a limited memory (much smaller than the input size which is $O\left(n^{2}\right)$ for a graph problem). In recent years, graph streaming algorithms and lower bounds for numerous problems have been studied extensively. In particular, we now have a relatively clear picture of the powers and limitations of single-pass algorithms. With a rather gross oversimplification, this can be stated as follows:

- The exact variant of most graph problems of interest are intractable: There are $\Omega\left(n^{2}\right)$ space lower bounds for maximum matching and minimum vertex cover [48,54], (directed) reachability and topological sorting [29,48,60], shortest path and diameter [48,49], minimum or maximum cut [89], maximal independent set $[7,38]$, dominating set $[8,46]$, and many others.

- On the other hand, approximate variants of many graph problems are tractable: There are $\widetilde{O}(n):=O(n \cdot \operatorname{polylog}(n))$ space algorithms (often referred to as semi-streaming algorithms) for approximate (weighted) matching and vertex cover $[41,47,48,79]$, spanner computation and approximation for distance problems $[17,44,45,49]$, cut or spectral sparsifiers and approximation for cut problems $[1,3,65,67]$, large independents sets [38,57], graph coloring [7,21], and approximate dominating set $[8,46]$, among others ${ }^{1}$.

Recent years have also witnessed a surge of interest in designing multi-pass graph streaming algorithms (see, e.g. [2, 18, 20, 29, 32, 39, 43, 48, 52, 62, 64, 66, 70, 73, 75, 84]); see, e.g., [48, 74] for discussions on practical applications of multi-pass streaming algorithms in particular in obtaining I/O-efficiency. These results suggest that allowing even just one more pass over the input greatly enhances the capability of the algorithms. For instance, while computing the exact global or $s$ - $t$ minimum cut in undirected graphs requires $\Omega\left(n^{2}\right)$ space in a single pass [89], perhaps surprisingly, one can solve both problems in only two passes with $\widetilde{O}(n)$ and $\widetilde{O}\left(n^{5 / 3}\right)$ space, respectively [82] (see also [76] for an $O(\log n)$-pass algorithm for weighted minimum cut). Qualitatively similar separations are known for numerous other problems such as triangle counting [27,39] (with two passes), approximate matching $[52,54,64,73]$ (with $O(1)$ passes), maximal independent set $[7,38,53]$ (with $O(\log \log n)$ passes), approximate dominating set [8,31,58] (with $O(\log n)$ passes), and exact shortest path $[32,49]$ (with $O(\sqrt{n})$ passes).

Despite this tremendous progress, the general picture for the abilities and limitations of multipass algorithms is not so clear even when we focus on two-pass algorithms. What other problems beside minimum cut admit non-trivial two-pass streaming algorithms? For instance, can we obtain similar results for directed versions of these problems? What about closely related problems such as maximum bipartite matching or not-so-related problems such as shortest path? Currently, none of these problems admit any non-trivial two-pass streaming algorithm, while known lower bounds only rule out algorithms with $o\left(n^{7 / 6}\right)$ space [29,49,56] leaving a considerable gap between upper and lower bounds (see [6] for a discussion on the current landscape of multi-pass graph streaming lower bounds and the challenges in improving them).

\subsection{Our Contributions}

We present near-quadratic space lower bounds for two-pass streaming algorithms for several fundamental graph problems including reachability, bipartite matching, and shortest path.

\footnotetext{
${ }^{1}$ It should be noted that, in contrast, determining the best approximation ratio possible for many of these problems have remained elusive and is an active area of research.
} 
Reachability and related problems in directed graphs. We prove the following lower bound for the reachability problem in directed graphs.

Result 1 (Formalized in Theorem 4). Any two-pass streaming algorithm (deterministic or randomized) that given an $n$-vertex directed graph $G=(V, E)$ with two designated vertices $s, t \in V$ can determine whether or not $s$ can reach $t$ in $G$ requires $\Omega\left(\frac{n^{2}}{2^{\Theta(\sqrt{\log n})}}\right)$ space.

The reachability problem is one of the earliest problems studied in the graph streaming model [60]. Previously, Henzinger et al. [60] and Feigenbaum et al. [49] proved an $\Omega\left(n^{2}\right)$ space lower bound for this problem for single-pass algorithms, and Guruswami and Onak [56] gave an $\widetilde{\Omega}_{p}\left(n^{1+1 /(2 p+2)}\right)$ lower bound for $p$-pass algorithms which translates to $\widetilde{\Omega}\left(n^{7 / 6}\right)$ space for two-pass algorithms; this lower bound was recently extended to random-order streams by Chakrabarti et al. [29]. Note that the undirected version of this problem has a simple $O(n \log n)$ space algorithm in one pass by maintaining a spanning forest of the input graph (see, e.g. [48]).

Using standard reductions, our results in this part can be extended to several other related problems on directed graphs such as estimating number of vertices reachable from a given source or approximating minimum feedback arc set, studied in [60] and [29], respectively.

Matching and cut problems. We have the following lower bound for bipartite matching.

Result 2 (Formalized in Theorem 5). Any two-pass streaming algorithm (deterministic or randomized) that given an n-vertex undirected bipartite graph $G=(L \sqcup R, E)$ can determine whether or not $G$ has a perfect matching requires $\Omega\left(\frac{n^{2}}{2^{\Theta(\sqrt{\log n})}}\right)$ space.

Maximum matching problem is arguably the most studied problem in the graph streaming model. However, the main focus on this problem so far has been on approximation algorithms and not much is known for exact computation of this problem, beside that it can be done in $\widetilde{O}\left(k^{2}\right)$ space in a single pass where $k$ is size of the maximum matching [36] (for the perfect matching problem, this gives an $O\left(n^{2}\right)$ space algorithm which is the same as storing the entire input). Previously, Feigenbaum et al. [48] and Chitnis et al. [37] proved an $\Omega\left(n^{2}\right)$ space lower bound for singlepass algorithms for this problem and Guruswami and Onak [56] extended the lower bound to $\widetilde{\Omega}_{p}\left(n^{1+1 /(2 p+2)}\right)$ for $p$-pass algorithms.

Both the perfect matching problem and the $s$ - $t$ reachability problem are simpler versions of the $s-t$ minimum cut problem in directed graphs. As such, our lower bounds imply that even though the $s$ - $t$ minimum cut problem can be solved in undirected graphs in $\widetilde{O}\left(n^{5 / 3}\right)$ space and two passes [82], its directed version requires $n^{2-o(1)}$ space in two passes (for any multiplicative approximation). Previously, Assadi et al. [6] proved a lower bound of $\Omega\left(n^{2} / p^{5}\right)$ for $p$-pass algorithms for the weighted $s$ - $t$ minimum cut problem (with exponential-in- $p$ weights); for the unweighted problem, the previous best lower bound was still $\widetilde{\Omega}_{p}\left(n^{1+1 /(2 p+2)}\right)$.

Shortest path problem. Finally, we also prove a lower bound for the shortest path problem.

Result 3 (Formalized in Theorem 6). Any two-pass streaming algorithm (deterministic or randomized) that given an undirected graph $G=(V, E)$ and two designated vertices $s, t \in V$, can output the length of the shortest s-t-path in $G$ requires $\Omega\left(\frac{n^{2}}{2^{\Theta(\sqrt{\log n})}}\right)$ space. The lower bound continues to hold even for approximation algorithms with approximation ratio better than 9/7. 
Shortest path problem have also been extensively studied in graph streaming literature. For single-pass streams, the focus has been on maintaining spanners (subgraphs that preserve pairwise distances approximately) which allow for obtaining algorithms with different space-approximation tradeoffs $[17,44,45,49]$ (starting from 2-approximation in $O\left(n^{3 / 2}\right)$ space to $O(\log n)$ approximation in $O(n)$ space), which are known to be almost tight [49]. For multi-pass algorithms, $\widetilde{O}(n)$ space algorithms are known for $(1+\varepsilon)$-approximation with poly $(\log n, 1 / \varepsilon)$ passes [18,59], and exact algorithms with $O(\sqrt{n})$ passes [32]. On the lower bound front, an $\Omega\left(n^{2}\right)$ space lower bound is known for single-pass algorithms [49] and $\widetilde{\Omega}_{p}\left(n^{1+1 / 2 p+2}\right)$ for $p$-pass algorithms [56]) (for exact answer or even some small approximation $\approx(2 p+4) /(2 p+2))$; a stronger lower bound of $\Omega\left(n^{1+1 / 2 p}\right)$ was proven earlier in [49] for algorithms that need to find the shortest path itself.

Our results show that a wide range of graph problems including directed reachability, cut and matching, and shortest path problems, admit essentially no non-trivial two-pass streaming algorithms: modulo the $n^{o(1)}$-term in our bounds, the best one could do to is to simply store the entire stream in $O\left(n^{2}\right)$ space and solve the problem at the end using any offline algorithm.

\subsection{Our Techniques}

We prove our main lower bound for the $s$ - $t$ reachability problem; the other lower bounds then follow easily from this using standard ideas.

It helps to start the discussion with the lower bounds in $[29,49,56]$. These lower bounds work with random graphs wherein $s$ can reach $\Theta(\sqrt{n})$ random vertices $S$ and $t$ is independently reachable from $\Theta(\sqrt{n})$ random vertices $T$; thus, by Birthday Paradox, $s$ - $t$ reachability can have either answer with constant probability. One then shows that to determine this, the algorithm needs to "find" $S$ and $T$ explicitly. The final part is then to use ideas from pointer chasing problems $[28,77,78,80,88]$ to prove a lower bound for this task. The particular space-pass tradeoff is then determined as follows: $(i)$ as a streaming algorithm can find the $p$-hop neighborhood of $s$ and $t$ in $p$ passes (by BFS), $S$ and $T$ need to be $(p+1)$-hop away from $s$ and $t$; $(i i)$ as we are working with random graphs, to achieve the bound of $O(\sqrt{n})$ on size of $S$ and $T$, we need the degree of the graph to be $O\left(n^{1 / 2(p+1)}\right)$, leading to an $O\left(n^{1+1 /(2 p+2)}\right)$ space lower bound for $p$-pass algorithms. We note that the limit of these approaches based on random graphs seem to be $\widetilde{O}\left(n^{3 / 2}\right)$; see [29, Section 5.2].

Our lower bound takes a different route and works with "more structured" graphs. We start with proving a single-pass streaming lower bound for an "algorithmically easier" variant of the reachability problem. In this problem, we are promised that $s$ can reach a unique vertex $s^{\star}$ chosen uniformly at random from a set $U$ of $n^{1-o(1)}$ vertices and the goal is to "find" this vertex. Previous lower bounds $[29,49,56]$ already imply that if our goal was to determine the identity of $s^{\star}$ exactly, we need $\Omega\left(n^{2}\right)$ space. In this paper, we prove a stronger lower bound that an $n^{2-o(1)}$-space singlepass algorithm essentially cannot even change the distribution of $s^{\star}$ from uniform over $U$. The proof of this part is based on information theoretic arguments that rely on "embedding" multiple instances of the set intersection problem (see Section 3) inside a Ruzsa-Szemerédi (RS) graph (see Section 2.2), and proving a new lower bound for the set intersection problem.

We remark that our new lower bound for set intersection is related to the recent lower bound of [6] with a subtle technical difference that is explained in Section 3 and Remark 5.7. We also note that RS graphs have been used extensively for proving graph streaming lower bounds $[9,10,38,54$, 64,69] starting from [54], but this is their first application to the $s$ - $t$ reachability problem.

In the next part of the argument, we construct a family of graphs in which the $s$ - $t$ reachability is determined by existence of a single edge $\left(s^{\star}, t^{\star}\right)$ in the graph, where $s^{\star}$ is the unique vertex reachable from $s$ in a large set $U$ and $t^{\star}$ is the unique vertex that can reach $t$ in a large set $W$ 
(see Figure 2 for an illustration). By exploiting our lower bound in the first part, we show that a $n^{2-o(1)}$-space algorithm cannot properly "find" the pairs $s^{\star}$ and $t^{\star}$ in the first pass. We then argue that this forces the algorithm to effectively "store" all the edges between $U$ and $W$ in the second pass to determine if $\left(s^{\star}, t^{\star}\right)$ is an edge of the graph, leading to an $n^{2-o(1)}$ space lower bound.

Remark (More than two passes?). The intermediate "simpler" problem we considered in our proofs (part one above) is only hard in one pass (see Section 4) and thus our lower bound proof does not directly go beyond two passes. However, it appears that our techniques can be extended to multi-pass algorithms to prove lower bounds of the type $n^{1+\Omega(1 / p)}$ space for p-pass algorithms which are slightly better in terms of dependence on $p$ in the exponent compared to [29,49,56]. Nevertheless, as unlike the case for two-pass algorithms, it is no longer clear whether such bounds are the "right" answer to the problems at hand, we opted to not pursue this direction in this paper.

\subsection{Subsequent Work}

Since the conference publication of this work in [14], our result has inspired several follow-ups. Firstly, [33] significantly strengthened our approach to prove $n^{2-o(1)}$-space lower bounds for the problems considered in this paper in any $o(\sqrt{\log n})$ passes. In a nutshell, their techniques can be seen as recursive construction that can "hide" a large subset of vertices from streaming algorithms as opposed to only a pair of vertices $s^{\star}, t^{\star}$ discussed in our techniques. Moreover, [5] built on the ideas in our work as well as [33] to prove a two-pass lower bound for approximating matchings up to some (small) constant factor (see also [71] that give a two-pass lower bound for a restricted family of algorithms that only compute a greedy matching in their first pass but then can be arbitrary in their second pass). Finally, [34] also used similar ideas to prove two-pass lower bounds for performing random walks in directed graphs.

In general, there has been a rapidly growing body of work on multi-pass graph streaming lower bounds in the last couple of years [5,11-14,29,33-35,68], and we refer the interested reader to these papers for more details.

\section{Preliminaries}

Notation. For any integer $t \geqslant 1$, we use $[t]:=\{1, \ldots, t\}$. For any $k$-tuple $X=\left(X_{1}, \ldots, X_{k}\right)$ and integer $i \in[k]$, we define $X^{<i}:=\left(X_{1}, \ldots, X_{i-1}\right)$.

Throughout the paper, we use 'sans serif' letters to denote random variables (e.g., A), and the corresponding normal letters to denote their values (e.g. A). For brevity and to avoid the clutter in notation, in conditioning terms which involve assignments to random variables, we directly use the value of the random variable (with the same letter), e.g., write $\mathrm{B} \mid A$ instead of $\mathrm{B} \mid \mathrm{A}=A$.

For random variables $A, B$, we use $\mathbb{H}(A)$ and $\mathbb{I}(A ; B):=\mathbb{H}(A)-\mathbb{H}(A \mid B)$ to denote the Shannon entropy and mutual information, respectively. Moreover, for two distributions $\mu, \nu$ on the same support, $\|\mu-\nu\|_{\text {tvd }}$ denotes the total variation distance, and $\mathbb{D}(\mu \| \nu)$ is the KL-divergence. A summary of basic information theory facts that we use in our proofs appear in Appendix A.

Concentration bounds: We will use the following variant of Chernoff bound (see, e.g. [42]).

Proposition 2.1 (Chernoff bound). Let $\mathrm{X}_{1}, \ldots, \mathrm{X}_{n}$ be $n$ independent random variables with values in $[0,1]$ and $\mathbf{X}:=\sum_{i=1}^{n} \mathbf{X}_{i}$. Then, for any $b>0$,

$$
\operatorname{Pr}(|\mathbf{X}-\mathbb{E}[\mathbf{X}]| \geqslant b) \leqslant 2 \cdot \exp \left(-\frac{b^{2}}{2 n}\right)
$$




\subsection{Communication Complexity and Information Complexity}

We work with the two-party communication model of Yao [86] (with some slightly non-standard aspects mentioned in Section 5). See the excellent textbook by Kushilevitz and Nisan [72] for an overview of communication complexity.

Let $P: \mathcal{X} \times \mathcal{Y} \rightarrow \mathcal{Z}$ be a relation. Alice receives an input $X \in \mathcal{X}$ and Bob receives $Y \in \mathcal{Y}$, where $(X, Y)$ are chosen from a distribution $\mathcal{D}$ over $\mathcal{X} \times \mathcal{Y}$. We allow players to have access to both public and private randomness. They communicate with each other by exchanging messages according to some protocol $\pi$. Each message in $\pi$ depends only on the private input and random bits of the player sending the message, the already communicated messages, and the public randomness. At the end, one of the players outputs an answer $Z$ such that $Z \in P(X, Y)$. For any protocol $\pi$, we use $\Pi:=\Pi(X, Y)$ to denote the messages and the public randomness used by $\pi$ on the input $(X, Y)$.

We now define two measures of "cost" of a protocol.

Definition 2.2 (Communication cost). The communication cost of a protocol $\pi$, denoted by $\mathrm{CC}(\pi)$, is the worst-case length of the messages communicated between Alice and Bob in the protocol.

Definition 2.3 (Information cost). The (internal) information cost of a protocol $\pi$, when the inputs $(X, Y)$ are drawn from a distribution $\mathcal{D}$, is $\mathrm{IC}_{\mathcal{D}}(\pi):=\mathbb{I}(\Pi ; \mathrm{X} \mid \mathrm{Y})+\mathbb{I}(\Pi ; \mathrm{Y} \mid \mathrm{X})$.

The internal information cost (introduced by [16]; see also [15, 16, 22, 25, 30]) measures the average amount of information each player learns about the input of the other player by observing the transcript of the protocol. As each bit of communication cannot reveal more than one bit of information, the internal information cost of a protocol lower bounds its communication cost [25].

Communication complexity and streaming. There is a standard connection between the communication cost of any protocol $\pi$ for a communication problem $P(X, Y)$ and the space of any streaming algorithm that can determine the value of $P(X, Y)$ on a stream $X \circ Y$ (see Proposition 6.1); we use this connection to establish our streaming lower bounds.

\subsection{Ruzsa-Szemerédi Graphs}

A graph $G^{\mathrm{RS}}=(V, E)$ is a called an $(r, t)$-Ruzsa-Szemerédi graph (RS graph for short) iff its edgeset $E$ can be partitioned into $t$ induced matchings $M_{1}^{\mathrm{RS}}, \ldots, M_{t}^{\mathrm{RS}}$, each of size $r$. We further define an $(r, t)$-RS digraph as a directed bipartite graph $G^{\mathrm{RS}}=(L, R, E)$ obtained by directing every edge of a bipartite $(r, t)$-RS graph from $L$ to $R$.

We use the original construction of RS graphs due to Ruzsa and Szemerédi [83] based on the existence of large sets of integers with no 3-term arithmetic progression, proven by Behrend [19]. We note that there are multiple other constructions with different parameters (see, e.g. $[4,50,51,54]$ and references therein) but the following construction works best for our purpose.

Proposition 2.4 ([83]). For infinitely many integers $N$, there are $(r, t)-R S$ digraphs with $N$ vertices on each side of the bipartition and parameters $r=\frac{N}{e^{\Theta(\sqrt{\log N})}}$ and $t=N / 3$.

\section{A New Lower Bound for the Set Intersection Problem}

One key ingredient of our paper is a new lower bound for the set intersection problem, defined formally as follows.

Problem 1 (set-intersection). The set-intersection problem is a two-player communication problem in which Alice and Bob are given sets $A$ and $B$ from $[m]$, respectively, with the promise that there 
exists a unique element $e^{\star}$ such that $\left\{e^{\star}\right\}=A \cap B$. The goal is to find the target element $e^{\star}$ using back and forth communication (i.e., in the two-way communication model).

The set-intersection problem is closely related to the well-known set disjointness problem. It is in fact straightforward to prove an $\Omega(m)$ lower bound on the communication complexity of set-intersection using a simple reduction from the set disjointness problem. However, in this paper, we are interested in an algorithmically simpler variant of this problem which we define below.

Definition 3.1. Let $\mathcal{D}$ be a distribution of inputs $(A, B)$ for set-intersection (known to both players). A protocol $\pi$ internal $\varepsilon$-solves set-intersection over $\mathcal{D}$ iff at least one of the following holds:

$$
\underset{\Pi, A}{\mathbb{E}}\left\|\operatorname{dist}\left(\mathrm{e}^{\star} \mid \Pi, A\right)-\operatorname{dist}\left(\mathrm{e}^{\star} \mid A\right)\right\|_{\mathrm{tvd}} \geqslant \varepsilon \quad \text { or } \underset{\Pi, \mathrm{B}}{\mathbb{E}}\left\|\operatorname{dist}\left(\mathrm{e}^{\star} \mid \Pi, B\right)-\operatorname{dist}\left(\mathrm{e}^{\star} \mid B\right)\right\|_{\mathrm{tvd}} \geqslant \varepsilon,
$$

where all variables are defined with respect to the distribution $\mathcal{D}$ and the internal randomness of $\pi$ (recall that $\Pi$ includes the transcript and the public randomness).

Definition 3.1 basically states that a protocol can internal $\varepsilon$-solve the set-intersection problem iff the transcript of the protocol can change the distribution of the target element $e^{\star}$ from the perspective of Alice or Bob by at least $\varepsilon$ in the total variation distance on average.

Our definition is inspired but different from $\varepsilon$-solving in [6] (which we call external $\varepsilon$-solving to avoid ambiguity) which required the transcript to change the distribution of the target element by $\varepsilon$ from the perspective of an external observer (who only sees the transcript but not the inputs of players). More formally, external $\varepsilon$-solving of set-intersection over a distribution $\mu$, as defined in [6], requires the protocol $\pi$ to have the following property (compare this with Definition 3.1),

$$
\underset{\Pi}{\mathbb{E}}\left\|\operatorname{dist}\left(\mathrm{e}^{\star} \mid \Pi\right)-\operatorname{dist}\left(\mathrm{e}^{\star}\right)\right\|_{\mathrm{tvd}} \geqslant \varepsilon
$$

The previous work in [6] has shown that there is a distribution $\mu$ such that any protocol that external $\varepsilon$-solves set-intersection over $\mu$ has information cost $\Omega\left(\varepsilon^{2} \cdot m\right)$. This however does not imply a lower bound for the internal $\varepsilon$-solving problem. This is because, in principle, these two tasks can be different. For instance, $(i)$ a protocol that reveals the entire set of Alice, changes the distribution of target for Bob dramatically but not so much for an external observer; or (ii) a protocol that reveals all the elements that are neither in $A$ nor in $B$ changes the distribution of the target for an external observer by a lot but does not change the distribution for either of the players at all.

We prove the following lower bound on the information cost of internal $\varepsilon$-solving of set-intersection.

Theorem 1. There is a distribution $\mathcal{D}_{\mathrm{SI}}$ for set-intersection over the universe $[m]$ such that:

(i) For any $A$ or $B$ sampled from $\mathcal{D}_{\mathrm{SI}}$, both $\operatorname{dist}\left(\mathrm{e}^{\star} \mid A\right)$ and $\operatorname{dist}\left(\mathrm{e}^{\star} \mid B\right)$ are uniform distributions on $A$ and $B$, each of size $m / 4$, respectively.

(ii) For any $\varepsilon \in(0,1)$, any protocol $\pi$ that internal $\varepsilon$-solves the set-intersection problem over the distribution $\mathcal{D}_{\mathrm{SI}}$ (Definition 3.1) has internal information cost $\mathrm{IC}_{\mathcal{D}_{\mathrm{SI}}}(\pi)=\Omega\left(\varepsilon^{2} \cdot m\right)$.

In the rest of this section, we give a simple proof of Theorem 1 by relating it to known results for exact solving of the set-intersection problem (our proof idea can also be used to give a much simpler proof of the lower bound of [6] for the external $\varepsilon$-solving of set-intersection). However, the reader may skip this section entirely and jump to the next one for the proof of our graph streaming lower bounds directly (the only result used from this section that is used outside is Theorem 1). 


\subsection{The Lower Bound for $\varepsilon$-Internal Solving of Set Intersection Background on Set Intersection}

Consider the following distribution:

Distribution $\mathcal{D}_{\mathrm{SI}}$. An input distribution $(A, B)$ to set-intersection over the universe $[\mathrm{m}]$.

(i) Sample two disjoint sets $\tilde{A}$ and $\tilde{B}$ of size $m / 4-1$ each uniformly at random from $[m]$.

(ii) Sample $e^{\star} \in[m] \backslash \tilde{A} \cup \tilde{B}$ uniformly at random and let $A:=\tilde{A} \cup\left\{e^{\star}\right\}$ and $B:=\tilde{B} \cup\left\{e^{\star}\right\}$.

It is well-known that $\mathcal{D}_{\mathrm{SI}}$ is a "hard" distribution for set-intersection [81]. In particular, the following result follows from the lower bound of [61].

Proposition 3.2 (cf. [61]). Any protocol $\pi$ that finds the target element in set-intersection over $\mathcal{D}_{\mathrm{SI}}$ with probability of success at least $2 / 3$ has information cost $\mathrm{IC}_{\mathcal{D}_{\mathrm{SI}}}(\pi)=\Omega(m)$.

A remark on the origin of Proposition 3.2 is in order. Firstly, if one only cares about proving a communication cost lower bound for set-intersection, then the proposition follows from the classical results on the communication complexity of the set disjointness problem [15,63,81]. At the same time, the lower bound on the information cost in Proposition 3.2 does not follow from the standard information cost lower bounds for set disjointness (see, e.g. [15,23,24,85]). This is because all aforementioned works bound the information cost of protocols for set disjointness on non-intersecting distributions, while $\mathcal{D}_{\mathrm{SI}}$ is crucially intersecting (one can fix this however by applying the recent result of [55], itself based on [26]). As such, one needs to apply the result of [61] on the information cost of set disjointness protocols on intersecting distributions (see [6] for details).

\section{Proof of Theorem 1}

Let $\pi_{\mathrm{SI}}$ be any protocol that internal $\varepsilon$-solves set-intersection over $\mathcal{D}_{\mathrm{SI}}$ and without loss of generality, let us assume it does so by changing the distribution of $e^{\star}$ from the perspective of Alice, i.e.,

$$
\underset{\Pi, \mathrm{A}}{\mathbb{E}}\left\|\operatorname{dist}\left(\mathrm{e}^{\star} \mid \Pi, A\right)-\operatorname{dist}\left(\mathrm{e}^{\star} \mid A\right)\right\|_{\mathrm{tvd}} \geqslant \varepsilon
$$

Define the following two constants:

$$
\begin{aligned}
& \gamma_{1}:=\text { minimum of } 1 / 100 \text { and the constant of } \Omega \text {-notation of Proposition 3.2, } \\
& \gamma_{2}:=100 \cdot \log \left(1 / \gamma_{1}\right) \text { for the constant } \gamma_{1} \text { above. }
\end{aligned}
$$

We design a protocol $\pi$ based on $\pi_{\mathrm{SI}}$ that finds the target element $e^{\star}$ of a given instance $(A, B)$ of set-intersection sampled from $\mathcal{D}_{\mathrm{SI}}$ with probability at least $2 / 3$. The general idea of the protocol is to use a "self-reducibility" property by turning each fixed instance of $\mathcal{D}_{\mathrm{SI}}$ to many independent instances sampled uniformly from $\mathcal{D}_{\mathrm{SI}}$ and run the protocol $\pi_{\mathrm{SI}}$ on each one. Alice then collects the elements that she deems to be "more likely" to be the target element in each of these instances into a set $T$ much smaller than $A$, and communicates $T$ directly to Bob. Our analysis uses the fact that the distribution of $e^{\star}$ from the perspective of Alice in each of these instances was sufficiently far from uniform and prove that this smaller set $T$ still should contain the target element $e^{\star}$ with a constant probability. 
Protocol $\pi$. A protocol for (exact) solving set-intersection on $\mathcal{D}_{\mathrm{SI}}$ using $\pi_{\mathrm{SI}}$ as a black-box.

(i) Define the parameters:

$$
k:=\frac{32}{\varepsilon^{2}} \cdot \ln \left(\frac{100 \gamma_{2}}{\gamma_{1}}\right), \quad t:=\frac{\gamma_{1}}{\gamma_{2}} \cdot \frac{m}{2}, \quad \tau:=\left(\frac{1}{2}+\frac{\varepsilon}{4}\right) \cdot k
$$

(ii) For $i=1$ to $k$ times:

(a) Sample a permutation $\sigma_{i}$ of $[m]$ uniformly at random using public randomness.

(b) Run $\pi_{\mathrm{SI}}$ on the inputs $\left(\sigma_{i}(A), \sigma_{i}(B)\right)$ (where for $S \in\{A, B\}, \sigma(S):=\{\sigma(i) \mid i \in S\}$ ).

(c) Let $\Pi_{i}$ be the transcript of $\pi_{\mathrm{SI}}$. Alice computes $\operatorname{dist}\left(e^{\star} \mid \Pi_{i}, \sigma_{i}(A)\right)$ and let $S_{i}$ be the top half of elements in $A$ with largest probability in this distribution.

(iii) For any element $e \in A$, Alice computes $c_{e}$ as the number of $i \in[k]$ such that $\sigma_{i}(e)$ appears in $S_{i}$. Let $T$ be the set of elements $e \in A$ where $c_{e}>\tau$.

(iv) If $|T|>t$, Alice reports 'fail'. Otherwise, Alice communicates the set $T$ directly to Bob (appended with arbitrary elements from $A$ until its size reaches exactly $t$ if it is smaller). Bob returns the element in $B \cap T$ or reports 'fail' if no element was found.

Observation 3.3. The distribution of each $\left(\sigma_{i}(A), \sigma_{i}(B)\right)$ is $\mathcal{D}_{\mathrm{SI}}$, and $\left\{\left(\sigma_{i}(A), \sigma_{i}(B)\right)\right\}_{i \in[k]}$ are mutually independent.

To see the proof of Observation 3.3 consider the following alternative way of sampling from the distribution $\mathcal{D}_{\mathrm{SI}}$ : Let $A=\{1, \ldots, m / 4\}$ and $B=\{m / 4, \ldots,(m / 2)-1\}$, and then apply a random permutation $\sigma$ and return $\sigma(A), \sigma(B)$. Under this view, Observation 3.3 is immediate.

Observation 3.3 identifies the role of $\sigma_{i}$ 's in the protocol - they basically "re-randomize" the input instance so that we can try an independent version of $\pi_{\mathrm{SI}}$ on the original input multiple times.

The following two claims are the key parts of the proof.

Claim 3.4. For any $i \in[k], \operatorname{Pr}\left(\sigma_{i}\left(e^{\star}\right) \in S_{i}\right) \geqslant \frac{1+\varepsilon}{2}$.

Proof. Considering Observation 3.3, any choice of $\Pi_{i}, \sigma_{i}(A)$, fixes $\operatorname{dist}\left(\sigma_{i}\left(e^{\star}\right) \mid \Pi_{i}, \sigma_{i}(A)\right)$ and the distribution of $\sigma_{i}\left(e^{\star}\right)$ over $\sigma_{i}(A)$ now is exactly this. At the same time, $\operatorname{dist}\left(\sigma_{i}\left(e^{\star}\right) \mid \sigma_{i}(A)\right)$ is uniform over $\sigma_{i}(A)$ by construction of $\mathcal{D}_{\mathrm{SI}}$. We can now use Proposition A.8 (on probability mass of larger half of distribution based on the total variation distance from uniform) to obtain,

$$
\begin{aligned}
\operatorname{Pr}\left(\sigma_{i}\left(e^{\star}\right) \in S_{i}\right) & =\underset{\Pi_{i}, \sigma_{i}(A)}{\mathbb{E}}\left[\operatorname{Pr}\left(\sigma_{i}\left(e^{\star}\right) \in S_{i} \mid \Pi_{i}, \sigma_{i}(A)\right)\right] \\
& \geqslant \underset{\Pi_{i}, \sigma_{i}(A)}{\mathbb{E}}\left[\frac{1}{2}+\frac{1}{2} \cdot\left\|\operatorname{dist}\left(\sigma_{i}\left(e^{\star}\right) \mid \Pi_{i}, \sigma_{i}(A)\right)-\operatorname{dist}\left(\sigma_{i}\left(e^{\star}\right) \mid \sigma_{i}(A)\right)\right\|_{\mathrm{tvd}}\right]
\end{aligned}
$$

(by Proposition A.8 and the choice of $S_{i}$ in the protocol)

$\geqslant \frac{1}{2}+\frac{\varepsilon}{2}$

where the final equation is by the guarantee of $\pi_{\mathrm{SI}}$ for internal $\varepsilon$-solving in Eq (2). 
Claim 3.5. For any $i \in[k]$ and $e \neq e^{\star} \in A, \operatorname{Pr}\left(\sigma_{i}(e) \in S_{i}\right) \leqslant \frac{1}{2}+\frac{1}{|A|}$.

Proof. Consider any fixing of $\left(\sigma_{i}(A), \sigma_{i}(B)\right)$. Over the randomness of $\sigma_{i}$, any element $e \neq e^{\star} \in A$ is mapped to $\sigma_{i}(A) \backslash \sigma_{i}\left(e^{\star}\right)$ uniformly at random even conditioned on $\sigma_{i}(A), \sigma_{i}(B)$ (because $\left.\sigma_{i}(e) \notin B\right)$. At the same time, fixing of $\left(\sigma_{i}(A), \sigma_{i}(B)\right)$ also fixes $\Pi_{i}$ and subsequently the set $S_{i}$. As such,

$$
\operatorname{Pr}\left(\sigma_{i}(e) \in S_{i}\right) \leqslant \frac{\left|S_{i}\right|}{|A|-1}=\frac{1}{2} \cdot \frac{1}{1-\frac{1}{|A|}} \leqslant \frac{1}{2} \cdot\left(1+\frac{2}{|A|}\right)=\frac{1}{2}+\frac{1}{|A|},
$$

concluding the proof.

Claims 3.4 and 3.5 imply that $e^{\star}$ tends to appear more frequently in the sets $S_{i}$ for $i \in[k]$ compared to other elements $e \in A$. Hence, by picking the threshold $\tau$ for adding the elements into $T$ carefully, we are able to ensure that $e^{\star} \in T$ with large probability, while not too many other elements belong to $T$ as well. We formalize this in the following claim whose proof is a basic application of Chernoff bound.

Claim 3.6. With probability at least $9 / 10, e^{\star} \in T$ and $|T| \leqslant t$.

Proof. For any element $e \in A$, let $X(e)$ denote the number indices $i \in[k]$ where $\sigma_{i}(e)$ appears in $S_{i}$, i.e., $X(e)=c_{e}$. By Claim 3.4, we have

$$
\mathbb{E}\left[X\left(e^{\star}\right)\right] \geqslant\left(\frac{1}{2}+\frac{\varepsilon}{2}\right) \cdot k=\tau+\frac{\varepsilon}{4} \cdot k . \quad \text { (by the choice of } \tau=\left(\frac{1}{2}+\frac{\varepsilon}{4}\right) \cdot k \text { ) }
$$

Moreover, by Observation 3.3, the choices across the $k$ instances $\left(\sigma_{i}(A), \sigma_{i}(B)\right)$ are independent. As such, by Chernoff bound (Proposition 2.1),

$$
\operatorname{Pr}\left(X\left(e^{\star}\right) \leqslant \tau\right) \leqslant \operatorname{Pr}\left(\left|X\left(e^{\star}\right)-\mathbb{E}\left[X\left(e^{\star}\right)\right]\right| \geqslant \frac{\varepsilon}{4} \cdot k\right) \leqslant 2 \cdot \exp \left(-\frac{\varepsilon^{2}}{32} \cdot k\right) \leqslant \frac{\gamma_{1}}{100 \cdot \gamma_{2}} \leqslant \frac{1}{100}
$$

(by the choice of $k=\frac{32}{\varepsilon^{2}} \cdot \ln \left(\frac{100 \cdot \gamma_{2}}{\gamma_{1}}\right)$ in $\pi$, and since $\gamma_{1} \leqslant 1$ and $\gamma_{2} \geqslant 1$ in Eq (3))

This means that $e^{\star} \in T$ with probability at least 99/100.

At the same time, for any $e \neq e^{\star}$, by Claim 3.5,

$$
\mathbb{E}[X(e)] \leqslant\left(\frac{1}{2}+\frac{4}{m}\right) \cdot k \leqslant \tau-\frac{\varepsilon}{4} \cdot k+1, \quad \text { (by the choice of } \tau=\left(\frac{1}{2}+\frac{\varepsilon}{4}\right) \cdot k \text { ) }
$$

where we assumed that $m>4 k$ as otherwise $\varepsilon^{2} \cdot m=\Theta(1)$ and thus Theorem 1 hold vacuously.

As before, by Chernoff bound,

$$
\operatorname{Pr}(X(e)>\tau) \leqslant \operatorname{Pr}\left(|X(e)-\mathbb{E}[X(e)]| \geqslant \frac{\varepsilon}{4} \cdot k\right) \leqslant \frac{\gamma_{1}}{100 \cdot \gamma_{2}} . \quad \quad \text { (as calculated above) }
$$

As such,

$$
\mathbb{E}|T| \leqslant 1+\sum_{e \neq e^{\star} \in A} \mathbb{E}[X(e)] \leqslant 1+\frac{m}{4} \cdot \frac{\gamma_{1}}{100 \cdot \gamma_{2}},
$$

and thus, by Markov bound, with probability less than $1 / 100,|T| \leqslant \frac{\gamma_{1}}{\gamma_{2}} \cdot \frac{m}{2}=t$, as desired. The claim now follows from a union bound. 
We need yet another claim that bounds the information cost of the protocol $\pi$. The proof uses the fact that Observation 3.3 ensures each $\left(\sigma_{i}(A), \sigma_{i}(B), \Pi_{i}\right)$ are distributed as in $\mathcal{D}_{\mathrm{SI}}$ (and hence we can bound its information cost) and the final set $T$ communicated by Alice is sufficiently small.

Claim 3.7. $\mathrm{IC}_{\mathcal{D}_{\mathrm{SI}}}(\pi) \leqslant k \cdot \mathrm{IC}_{\mathcal{D}_{\mathrm{SI}}}\left(\pi_{\mathrm{SI}}\right)+\frac{\gamma_{1}}{2} \cdot m$.

Proof. Let $\Pi_{1}, \ldots, \Pi_{k}$ and $\sigma_{1}, \ldots, \sigma_{k}$ denote the random variables for, respectively, the transcript of the protocol $\pi_{\mathrm{SI}}$ concatenated with its internal public randomness, and the random permutations in iterations 1 to $k$ of the for-loop in $\pi$. Additionally, let $\mathrm{T}$ be the random variable for the set $T$ communicated by Alice to Bob. By the definition of internal information cost and the chain rule of mutual information (Fact A.1-(4)): $I C_{\mathcal{D}_{\mathrm{SI}}}(\pi)$ is sum of the following three terms:

$$
\begin{aligned}
& \mathbb{I}\left(\sigma_{1}, \ldots, \sigma_{k} ; \mathrm{A} \mid \mathrm{B}\right)+\mathbb{I}\left(\sigma_{1}, \ldots, \sigma_{k} ; \mathrm{B} \mid \mathrm{A}\right) \\
& \mathbb{I}\left(\Pi_{1}, \ldots, \Pi_{k} ; \mathrm{A} \mid \mathrm{B}, \sigma_{1}, \ldots, \sigma_{k}\right)+\mathbb{I}\left(\Pi_{1}, \ldots, \Pi_{k} ; \mathrm{B} \mid \mathrm{A}, \sigma_{1}, \ldots, \sigma_{k}\right) ; \\
& \mathbb{I}\left(\mathrm{T} ; \mathrm{A} \mid \mathrm{B}, \Pi_{1}, \ldots, \Pi_{k}, \sigma_{1}, \ldots, \sigma_{k}\right)+\mathbb{I}\left(\mathrm{T} ; \mathrm{B} \mid \mathrm{A}, \Pi_{1}, \ldots, \Pi_{k}, \sigma_{1}, \ldots, \sigma_{k}\right) .
\end{aligned}
$$

We bound each term separately below.

Bounding Eq (4). This term is zero because the permutations are chosen independent of $(A, B)$ and thus the given mutual information term is zero by Fact A.1-(2).

Bounding Eq (5). Define $\Sigma:=\left(\sigma_{1}, \ldots, \sigma_{k}\right)$. We have,

$$
\begin{aligned}
\mathbb{I}\left(\Pi_{1}, \ldots, \Pi_{k} ; \mathrm{A} \mid \mathrm{B}, \Sigma\right)+\mathbb{I}\left(\Pi_{1}, \ldots, \Pi_{k} ; \mathrm{B} \mid \mathrm{A}, \Sigma\right) & =\sum_{i=1}^{k} \mathbb{I}\left(\Pi_{i} ; \mathrm{A} \mid \mathrm{B}, \Sigma, \Pi_{<i}\right)+\mathbb{I}\left(\Pi_{i} ; \mathrm{B} \mid \mathrm{A}, \Sigma, \Pi_{<i}\right) \\
\text { (by chain rule of mutual information (Fact A.1-(4))) } & \leqslant \sum_{i=1}^{k} \mathbb{I}\left(\Pi_{i} ; \mathrm{A} \mid \mathrm{B}, \sigma_{i}\right)+\mathbb{I}\left(\Pi_{i} ; \mathrm{B} \mid \mathrm{A}, \sigma_{i}\right)
\end{aligned}
$$

(as $\Pi_{i} \perp \Sigma_{<i}, \Pi_{<i} \mid \mathrm{A}, \mathrm{B}, \sigma_{i}$ since the conditioning fixes $\Pi_{i}$ and we can apply Proposition A.4)

$$
=\sum_{i=1}^{k} \mathbb{I}\left(\Pi_{i} ; \sigma_{i}(\mathrm{~A}) \mid \sigma_{i}(\mathrm{~B})\right)+\mathbb{I}\left(\Pi_{i} ; \sigma_{i}(\mathrm{~B}) \mid \sigma_{i}(\mathrm{~A})\right)
$$

(exactly as above as $\Pi_{i}$ only depends on the "inner" instance defined by $\sigma_{i}(A)$ and $\sigma_{i}(B)$ )

$$
=k \cdot \mathrm{IC}_{\mathcal{D}_{\mathrm{SI}}}\left(\pi_{\mathrm{SI}}\right) \text {. }
$$

(by Observation 3.3)

Bounding Eq (6). We use $\Sigma$ defined in the previous part here as well:

$$
\mathbb{I}\left(\mathrm{T} ; \mathrm{A} \mid \mathrm{B}, \Pi_{1}, \ldots, \Pi_{k}, \Sigma\right)+\mathbb{I}\left(\mathrm{T} ; \mathrm{B} \mid \mathrm{A}, \Pi_{1}, \ldots, \Pi_{k}, \Sigma\right)=\mathbb{I}(\mathrm{T} ; \mathrm{A} \mid \mathrm{B}, \Pi, \Sigma)
$$

(as A, $\Pi, \Sigma$ fixes $\mathrm{T}$ and hence second term is zero (Fact A.1-(2)))

$$
\leqslant \mathbb{H}(\mathrm{T} \mid \mathrm{B}, \Pi, \Sigma)
$$

(by definition of mutual information and non-negativity of entropy))

$$
\leqslant \mathbb{H}(\mathrm{T})
$$

(as conditioning cannot increase entropy (Fact A.1-(3) ))

$$
\leqslant \log \left(\begin{array}{c}
m \\
t
\end{array}\right)
$$

(as $\mathrm{T}$ is supported on $t$-subsets of $[m]$ and by Fact A.1-(1)) 


$$
\leqslant t \cdot \log \left(\frac{e \cdot m}{t}\right)
$$

(by standard upper bounds on binomial coefficients)

$$
\leqslant \frac{\gamma_{1}}{\gamma_{2}} \cdot \frac{m}{2} \cdot \log \left(\frac{2 e \cdot \gamma_{2}}{\gamma_{1}}\right)
$$

(by the choice of $t$ in the protocol $\pi$ )

$$
\leqslant \gamma_{1} \cdot m \cdot \frac{1}{100 \cdot \log \left(1 / \gamma_{1}\right)} \cdot \log \left(\frac{1}{\gamma_{1}^{3}}\right)
$$

(by the choice of $\gamma_{2}=100 \cdot \log \left(1 / \gamma_{1}\right) \leqslant 100 \cdot\left(1 / \gamma_{1}\right)$ and since $\gamma_{1} \leqslant 1 / 100$ in Eq (3))

$$
<\frac{\gamma_{1}}{2} \cdot m
$$

by the definition of $\gamma_{1}$ and Proposition 3.2. Putting the bounds in Eq (4), Eq (5), and Eq (6) for $\mathrm{IC}_{\mathcal{D}_{\mathrm{SI}}}(\pi)$ proves the claim.

We are now ready to conclude the proof of Theorem 1. Firstly, by Claim 3.6, the probability that the protocol $\pi$ finds the correct index $e^{\star}$ is at least $9 / 10$, because conditioned on the events in Claim 3.6, Alice simply sends all of $T$ to Bob which includes $e^{\star}$ and Bob will be able to output the answer. As such, by Proposition 3.2, we know that the information cost of $\pi$ has to be $\gamma_{1} \cdot m$ at least. Combining this with Claim 3.7 implies that

$$
\gamma_{1} \cdot m \leqslant \mathrm{IC}_{\mathcal{D}_{\mathrm{SI}}}(\pi) \leqslant k \cdot \mathrm{IC}_{\mathcal{D}_{\mathrm{SI}}}\left(\pi_{\mathrm{SI}}\right)+\frac{\gamma_{1}}{2} \cdot m
$$

and in turn results in

$$
\mathrm{IC}_{\mathcal{D}_{\mathrm{SI}}}\left(\pi_{\mathrm{SI}}\right) \geqslant \frac{\gamma_{1}}{2 k} \cdot m
$$

which is $\Omega\left(\varepsilon^{2} \cdot m\right)$ by the choice of $k$ and since $\gamma_{1}=\Theta(1)$ in Eq (3) (by Proposition 3.2). Theorem 1 now follows from this and the definition of the distribution $\mathcal{D}_{\mathrm{SI}}$ for part $(i)$.

\section{The Unique-Reach Communication Problem}

We now start with our main lower bounds. Define the following two-player communication problem.

Problem 2 (unique-reach). The unique-reach problem is defined as follows. Consider a digraph $G=(V, E)$ on $n$ vertices where $V:=\{s\} \sqcup V_{1} \sqcup V_{2} \sqcup V_{3}$ and any edge $(u, v) \in E$ is directed from $s$ to $V_{1}$ or some $V_{i}$ to $V_{i+1}$ for $i \in[2]$ (we refer to each $V_{i}$ as a layer). We are promised that there is a unique vertex $s^{\star}$ in the layer $V_{3}$ reachable from $s$.

Alice is given edges in $E$ from $V_{1}$ to $V_{2}$, denoted by $E_{A}$, and $B o b$ is given the remainder of the edges in $E$, denoted by $E_{B}$ (the partitioning of vertices of $V$ is known to both players). The goal for the players is to find $s^{\star}$ by Alice sending a single message to Bob (i.e., in the one-way communication model).

It is easy to prove a lower bound of $\Omega\left(n^{2}\right)$ on the one-way communication complexity of unique-reach using a reduction from the Index problem. It is also easy to see that this problem can be solved with $O(n \log n)$ bits of communication, if we allow Bob to send a single message to Alice: By the uniqueness promise on $s^{\star}$, no vertex with out-degree more than one in $V_{2}$ should be reachable from $s$ and thus Bob can communicate all the remaining edges in $E_{B}$ to Alice.

Nevertheless, in this paper, we are interested in an algorithmically simpler variant of this problem similar-in-spirit to $\varepsilon$-solving for set-intersection (Definition 3.1). 
Definition 4.1. Let $\mathcal{D}$ be any distribution of valid inputs $G=\left(V, E_{A} \sqcup E_{B}\right)$ for unique-reach (known to both players). We say that a protocol $\pi$ internal $\varepsilon$-solves unique-reach over $\mathcal{D}$ iff:

$$
\underset{\Pi, \mathrm{E}_{B}}{\mathbb{E}}\left\|\operatorname{dist}\left(\mathrm{s}^{\star} \mid \Pi, E_{B}\right)-\operatorname{dist}\left(\mathrm{s}^{\star} \mid E_{B}\right)\right\|_{\mathrm{tvd}} \geqslant \varepsilon
$$

where all variables are defined with respect to the distribution $\mathcal{D}$ and the internal randomness of $\pi$ (recall that $\Pi$ includes the transcript and the public randomness).

Definition 4.1 basically states that a protocol can internal $\varepsilon$-solve the problem iff the message sent from Alice can change the distribution of the unique vertex $s^{\star}$ from the perspective of Bob by at least $\varepsilon$ in the total variation distance (in expectation over Alice's message and Bob's input).

Our main theorem in this section is the following.

Theorem 2. There is a distribution $\mathcal{D}_{\mathrm{UR}}$ for unique-reach and an integer $b:=\frac{n}{2^{\Theta(\sqrt{\log n})} \text { with the }}$ following properties:

(i) For any $E_{B}$ sampled from $\mathcal{D}_{\mathrm{UR}}$, $\operatorname{dist}\left(\mathrm{s}^{\star} \mid E_{B}\right)$ is a uniform distribution over a subset $V_{3}^{\star}$ of $b$ vertices in the layer $V_{3}$ of the input graph;

(ii) for any $\varepsilon \in(0,1)$, any one-way protocol $\pi$ that internal $\varepsilon$-solves unique-reach over the distribution $\mathcal{D}_{\mathrm{UR}}$ (Definition 4.1) has communication cost $\mathrm{CC}(\pi)=\Omega\left(\varepsilon^{2} \cdot n \cdot b\right)$.

Proof of Theorem 2 is by a reduction from Theorem 1 using RS graphs (see Section 2.2).

\subsection{Distribution $\mathcal{D}_{\mathrm{UR}}$ in Theorem 2}

To continue, we need to set up some notation. Let $G^{\mathrm{RS}}=(L, R, E)$ be an $(r, t)$-RS digraph with induced matchings $M_{1}^{\mathrm{RS}}, \ldots, M_{t}^{\mathrm{RS}}$ as defined in Section 2.2. For each induced matching $M_{i}^{\mathrm{RS}}$, we assume an arbitrary ordering of edges $e_{i, 1}, \ldots, e_{i, r}$ in the matching and for each $j \in[r]$ denote $e_{i j}:=\left(u_{i j}, v_{i j}\right)$ for $u_{i j} \in L$ and $v_{i j} \in R$; moreover, we let $L\left(M_{i}^{\mathrm{RS}}\right):=\left\{u_{i 1}, \ldots, u_{i r}\right\}$ and $R\left(M_{i}^{\mathrm{RS}}\right):=$ $\left\{v_{i 1}, \ldots, v_{i r}\right\}$. Based on these, we have the following definition:

- For any matching $M_{i}^{\mathrm{RS}}$ and any set $S \subseteq[r]$, we define $M_{i}^{\mathrm{RS}} \mid S$ as the matching in $G^{\mathrm{RS}}$ consisting of the edges $e_{i j} \in M_{i}^{\mathrm{RS}}$ for all $j \in S$.

We are now ready to define our distribution. See Figure 1 for an illustration.

Distribution $\mathcal{D}_{\mathrm{UR}}$. An input distribution on graphs $G=\left(\{s\} \sqcup V_{1} \sqcup V_{2} \sqcup V_{3}, E_{A} \sqcup E_{B}\right)$.

(1) Let $G^{\mathrm{RS}}=\left(L, R, E^{\mathrm{RS}}\right)$ be a fixed $(r, t)$-RS digraph on $2 N$ vertices from Proposition 2.4 with parameters $r=\frac{N}{2^{\Theta(\sqrt{\log N})}}$, and $t=\frac{N}{3}$. We note that this graph is known to both players.

(2) Let $V_{1}=L=\left\{u_{1}, \ldots, u_{N}\right\}, V_{2}=R=\left\{v_{1}, \ldots, v_{N}\right\}$, and $V_{3}$ be $r$ new vertices $\left\{w_{1}, \ldots, w_{r}\right\}$.

(3) Sample $t$ independent instances $\left(S_{1}, T_{1}\right), \ldots,\left(S_{t}, T_{t}\right)$ of set-intersection on the universe $[r]$ from the distribution $\mathcal{D}_{\mathrm{SI}}$ in Theorem 1 .

(4) The input $E_{A}$ to Alice is $E_{A}:=\left(M_{1}^{\mathrm{RS}} \mid S_{1}\right) \cup \ldots \cup\left(M_{t}^{\mathrm{RS}} \mid S_{t}\right)$.

(5) Sample $i^{\star} \in[t]$ uniformly at random.

(6) The input $E_{B}$ to Bob is the set of edges $\left(s, u_{i^{\star} j}\right)$ for $j \in T_{i^{\star}}$ and $\left(v_{i^{\star} j}, w_{j}\right)$ for $j \in T_{i^{\star}}$. 


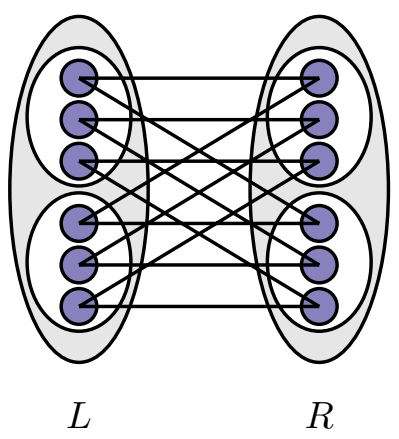

(a) A fixed $(3,4)$-RS digraph in the distribution.

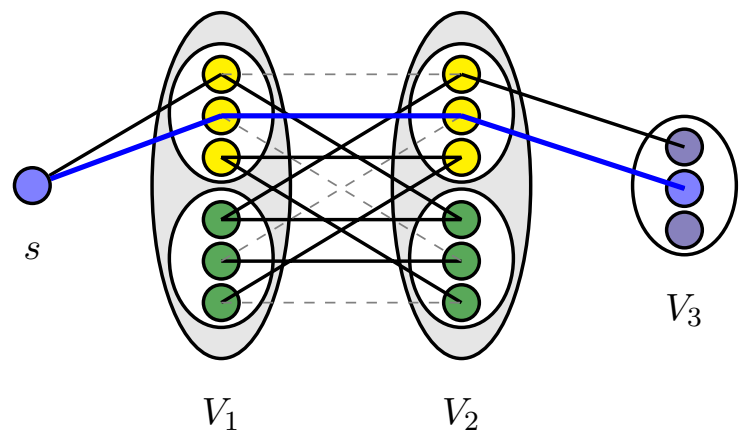

(b) The graph $G$ of $\mathcal{D}_{\text {UR }}$; dashed edges no longer belong to the graph, and yellow vertices are incident on $M_{i^{\star}}^{\mathrm{RS}}$.

Figure 1: An illustration of the input distribution $\mathcal{D}_{\mathrm{UR}}$. Here, directions of all edges are from left to right and hence omitted. The marked vertex (blue) in $V_{3}$ denotes the unique vertex $s^{\star}$ in this example along with the path connecting $s$ to $s^{\star}$.

Observation 4.2. Several observations are in order:

(i) For any $G \sim \mathcal{D}_{\mathrm{UR}}$, there is a unique vertex $s^{\star}$ reachable from $s$ in $V_{3}$. Moreover, $s^{\star}=w_{e^{\star}}$ where $e^{\star} \in[r]$ is the unique element in the intersection of $S_{i^{\star}}$ and $T_{i^{\star}}$.

(proof: $s^{\star}$ is reachable from $s$ through the path $s \rightarrow u_{i^{\star}, e^{\star}} \rightarrow v_{i^{\star}, e^{\star}} \rightarrow w_{e^{\star}}\left(=s^{\star}\right)$ and is the only such vertex by the uniqueness of $e^{\star}$ in $S_{i^{\star}} \cap T_{i^{\star}}$.)

(ii) For any $E_{B} \sim \mathcal{D}_{\mathrm{UR}}$, dist( $\left(\mathrm{s}^{\star} \mid E_{B}\right)$ is uniform over vertices $w_{j} \in V_{3}$ for $j \in T_{i^{\star}}$.

(proof: considering the one-to-one mapping between $s^{\star}$ and $w_{e^{\star}}$, and since $\operatorname{dist}\left(\mathrm{e}^{\star} \mid T_{i^{\star}}\right)$ is uniform over $T_{i^{\star}}$ by Theorem 1 , $\operatorname{dist}\left(\mathrm{s}^{\star} \mid E_{B}\right)$ is also uniform over $w_{j}$ for $j \in T_{i^{\star}}$.)

(iii) In $\mathcal{D}_{\mathrm{UR}}$, the index $i^{\star} \in[t]$ is independent of the sets $\left(S_{1}, T_{1}\right), \ldots,\left(S_{t}, T_{t}\right)$. Moreover, the pairs $\left(S_{1}, T_{1}\right), \ldots,\left(S_{t}, T_{t}\right)$ are mutually independent.

(iv) The input $E_{A}$ to Alice in $\mathcal{D}_{\mathrm{UR}}$ is uniquely determined by $S_{1}, \ldots, S_{t}$, and the input $E_{B}$ to Bob is determined by $i^{\star}$ and $T_{i^{\star}}$.

\subsection{Proof of Theorem 2}

Let $\pi_{\mathrm{UR}}$ be any one-way protocol that internal $\varepsilon$-solves unique-reach on the distribution $\mathcal{D}$ UR. We will prove that $\mathrm{CC}\left(\pi_{\mathrm{UR}}\right)=\Omega\left(\varepsilon^{2} \cdot r \cdot t\right)$ which proves Theorem 2 . The argument relies on the following two claims: $(i)$ internal $\varepsilon$-solving of unique-reach on $\mathcal{D}_{\text {UR }}$ is equivalent to internal $\varepsilon$ solving of set-intersection on $\mathcal{D}_{\mathrm{SI}}$ for the pair $\left(S_{i^{\star}}, T_{i^{\star}}\right)$; and $(i i)$ the information revealed by $\pi_{\mathrm{UR}}$ about the instance $\left(S_{i^{\star}}, T_{i^{\star}}\right)$ is at least $t$ times smaller than CC $\left(\pi_{\mathrm{UR}}\right)$. Having both these steps, we can then invoke Theorem 1 to conclude the proof.

We shall emphasize that this is not an immediate reduction from Theorem 1 as we are aiming to gain an additional factor of $t$ in the information cost lower bound for $\pi_{U R}$ compared to the lower bound for set-intersection. This part crucially relies on the fact that $\pi_{U R}$ is a one-way protocol and that index $i^{\star} \in[t]$ in the distribution is independent of Alice's input (and thus her message).

We now present the formal proof. Consider the following protocol $\pi_{\mathrm{SI}}$ for set-intersection on the distribution $\mathcal{D}_{\mathrm{SI}}$ using $\pi_{\mathrm{UR}}$ as a subroutine. 
Protocol $\pi_{\mathrm{SI}}$. Given an instance $(A, B) \sim \mathcal{D}_{\mathrm{SI}}$ on universe $[r]$, Alice and Bob do as follows:

(i) Alice and Bob sample $i^{\star} \in[t]$ using public randomness.

(ii) Alice sets $S_{i^{\star}}=A$ and samples the remaining sets $S_{i}$ for $i \neq i^{\star} \in[t]$ independently from $\mathcal{D}_{\mathrm{SI}}$ using private randomness (this is doable by part (iii) of Observation 4.2). This allows Alice to generate the set $E_{A}$ of edges for $\pi_{\mathrm{UR}}$ as in $\mathcal{D}_{\mathrm{UR}}$ (by part (iv) of Observation 4.2).

(iii) Bob sets $T_{i^{\star}}=B$ and creates the set of edges $E_{B}$ for $\pi_{\mathrm{UR}}$ as in $\mathcal{D}_{\mathrm{UR}}$ (again doable by part (iv) of Observation 4.2 as Bob also knows $i^{\star}$ ).

(iv) The players then run the protocol $\pi_{\mathrm{UR}}$ on the input $\left(E_{A}, E_{B}\right)$ with Alice sending the message in $\pi_{\text {UR }}$ to Bob.

We first prove the following claim.

Claim 4.3. $\pi_{\mathrm{SI}}$ internal $\varepsilon$-solves set-intersection on $\mathcal{D}_{\mathrm{SI}}$.

Proof. The distribution of instances $E_{A}, E_{B}$ created in the protocol $\pi_{\mathrm{SI}}$ (using the randomness of the input) is the same as $\mathcal{D}_{\mathrm{UR}}$. We can now examine whether $\pi_{\mathrm{SI}} \varepsilon$-solves set-intersection as follows:

$$
\underset{\Pi_{\mathrm{SI}}, \mathrm{B}}{\mathbb{E}}\left\|\operatorname{dist}\left(\mathrm{e}^{\star} \mid \Pi_{\mathrm{SI}}, B\right)-\operatorname{dist}\left(\mathrm{e}^{\star} \mid B\right)\right\|_{\mathrm{tvd}}=\underset{\Pi_{\mathrm{UR}}, i^{\star}, \mathrm{B}}{\mathbb{E}}\left\|\operatorname{dist}\left(\mathrm{e}^{\star} \mid \Pi_{\mathrm{UR}}, i^{\star}, B\right)-\operatorname{dist}\left(\mathrm{e}^{\star} \mid B\right)\right\|_{\mathrm{tvd}}
$$

(since $\Pi_{\mathrm{SI}}=\left(\Pi_{\mathrm{UR}}, \mathrm{i}^{\star}\right)$ as the only extra public randomness of $\pi_{\mathrm{SI}}$ over $\pi_{\mathrm{UR}}$ is the choice of $i^{\star}$ )

$$
=\underset{\Pi_{\mathrm{UR}}, \mathrm{E}_{B}}{\mathbb{E}}\left\|\operatorname{dist}\left(\mathrm{s}^{\star} \mid \Pi_{\mathrm{UR}}, E_{B}\right)-\operatorname{dist}\left(\mathrm{s}^{\star} \mid E_{B}\right)\right\|_{\mathrm{tvd}}
$$

since $\left(i^{\star}, B\right)$ uniquely identifies $E_{B}$ and vice versa, and since by Observation 4.2, modulo a renaming, we have $\operatorname{dist}\left(\mathrm{e}^{\star} \mid B\right)=\operatorname{dist}\left(s^{\star} \mid E_{B}\right)$. The last term above is now at least $\varepsilon$ since $\pi_{\mathrm{UR}}$ internal $\varepsilon$-solves set-intersection, which concludes the proof.

We now bound the internal information cost of $\pi_{\mathrm{SI}}$ which allows us to apply Theorem 1 and conclude the proof. The proof of this lemma is by a direct-sum style argument. We note that these arguments (based on information theory tools) are by now mostly standard in the literature.

Lemma 4.4. $\mathrm{IC}_{\mathcal{D}_{\mathrm{SI}}}\left(\pi_{\mathrm{SI}}\right) \leqslant \frac{1}{t} \cdot \mathrm{CC}\left(\pi_{\mathrm{UR}}\right)$.

Proof. By definition of the internal information cost,

$$
\mathrm{IC}_{\mathcal{D}_{\mathrm{SI}}}\left(\pi_{\mathrm{SI}}\right)=\mathbb{I}\left(\Pi_{\mathrm{SI}} ; \mathrm{A} \mid \mathrm{B}\right)+\mathbb{I}\left(\Pi_{\mathrm{SI}} ; \mathrm{B} \mid \mathrm{A}\right)=\mathbb{I}\left(\Pi_{\mathrm{SI}} ; \mathrm{A} \mid \mathrm{B}\right) \leqslant \mathbb{I}\left(\Pi_{\mathrm{SI}} ; \mathrm{A}\right)
$$

this is because $\pi_{\mathrm{SI}}$ is a one-way protocol and thus $\Pi_{\mathrm{SI}} \perp \mathrm{B} \mid \mathrm{A}$ and so we can apply Fact A.1-(2) for the last equality and Proposition A.4 for the final inequality. We now bound the RHS above:

$$
\mathbb{I}\left(\Pi_{S I} ; A\right)=\mathbb{I}\left(\Pi_{U R}, i^{\star} ; A\right)
$$

(as the only additional public randomness of $\pi_{\mathrm{SI}}$ over $\pi_{\mathrm{UR}}$ is the choice of $i^{\star}$ )

$$
\begin{array}{rlrl} 
& =\mathbb{I}\left(i^{\star} ; \mathrm{A}\right)+\mathbb{I}\left(\Pi_{\mathrm{UR}} ; \mathrm{A} \mid \mathrm{i}^{\star}\right) & \text { (by the chain rule of mutual information (Fact A.1- }(4))) \\
& =\mathbb{I}\left(\Pi_{\mathrm{UR}} ; \mathrm{A} \mid \mathrm{i}^{\star}\right) & \left(\text { as } \mathrm{i}^{\star} \perp \mathrm{A} \text { and so } \mathbb{I}\left(\mathrm{i}^{\star} ; \mathrm{A}\right)=0 \text { by Fact A.1- }(2)\right) \\
=\sum_{i^{\star}=1}^{t} \frac{1}{t} \cdot \mathbb{I}\left(\Pi_{\mathrm{UR}} ; \mathrm{A} \mid i^{\star}\right) & \quad\left(\text { as } i^{\star} \in[t]\right. \text { is chosen uniformly at random) }
\end{array}
$$




$$
\begin{aligned}
& =\frac{1}{t} \cdot \sum_{i^{\star}=1}^{t} \mathbb{I}\left(\Pi_{\mathrm{UR}} ; \mathrm{S}_{i^{\star}} \mid i^{\star}\right) \\
& =\frac{1}{t} \cdot \sum_{i^{\star}=1}^{t} \mathbb{I}\left(\Pi_{\mathrm{UR}} ; \mathrm{S}_{i^{\star}}\right)
\end{aligned}
$$

(as the input of Alice and her one-way message are independent of $i^{\star}=i^{\star}$ by Observation 4.2)

$$
\begin{array}{ll}
\leqslant \frac{1}{t} \cdot \sum_{i^{\star}=1}^{t} \mathbb{I}\left(\Pi_{\mathrm{UR}} ; \mathrm{S}_{i^{\star}} \mid \mathrm{S}^{<i^{\star}}\right) & \text { (by Proposition A.3 since } \left.\mathrm{S}_{i^{\star}} \perp \mathrm{S}^{<i^{\star}} \text { by Observation } 4.2\right) \\
=\frac{1}{t} \cdot \mathbb{I}\left(\Pi_{\mathrm{UR}} ; \mathrm{S}_{1}, \ldots, \mathrm{S}_{t}\right) . & \text { (by the chain rule of mutual information in Fact A.1-(4)) }
\end{array}
$$

We can now bound the $\mathbb{I}\left(\Pi_{U R} ; S_{1}, \ldots, S_{t}\right)$ term above by the communication cost of the protocol $\pi_{U R}$ as follows. Define $R$ as the public randomness of the protocol $\pi_{U R}$ and $\mathrm{M}$ as its message sent from Alice to Bob. We have,

$$
\mathbb{I}\left(\Pi_{U R} ; S_{1}, \ldots, S_{t}\right)=\mathbb{I}\left(M, R ; S_{1}, \ldots, S_{t}\right)=\mathbb{I}\left(R ; S_{1}, \ldots, S_{t}\right)+\mathbb{I}\left(M ; S_{1}, \ldots, S_{t} \mid R\right)
$$

(by the chain rule of mutual information (Fact A.1-(4)))

$$
=\mathbb{I}\left(\mathrm{M} ; \mathrm{S}_{1}, \ldots, \mathrm{S}_{t} \mid \mathrm{R}\right)
$$

(as $\mathrm{R}$ is independent of the input and thus the first term is zero by Fact A.1-(2))

$$
\leqslant \log |\operatorname{supp}(\mathrm{M})|=\mathrm{CC}\left(\pi_{\mathrm{UR}}\right) .
$$

Plugging the equations above together, we obtain that,

$$
\mathrm{IC}_{\mathcal{D}_{\mathrm{SI}}}\left(\pi_{\mathrm{SI}}\right) \leqslant \mathbb{I}\left(\Pi_{\mathrm{SI}} ; \mathrm{A}\right) \leqslant \frac{1}{t} \cdot \mathbb{I}\left(\Pi_{\mathrm{UR}} ; \mathrm{S}_{1}, \ldots, \mathrm{S}_{t}\right) \leqslant \frac{1}{t} \cdot \mathrm{CC}\left(\pi_{\mathrm{UR}}\right)
$$

proving the lemma.

We now conclude the proof of Theorem 2. By Claim 4.3, $\pi_{\mathrm{SI}}$ internal $\varepsilon$-solves set-intersection and thus by Theorem 1, we have $\mathrm{IC}_{\mathcal{D}_{\mathrm{SI}}}\left(\pi_{\mathrm{SI}}\right)=\Omega\left(\varepsilon^{2} \cdot r\right)$. Plugging in this bound in Lemma 4.4, we obtain that

$$
\mathrm{CC}\left(\pi_{\mathrm{UR}}\right)=\Omega\left(\varepsilon^{2} \cdot r \cdot t\right)=\Omega\left(\varepsilon^{2} \cdot \frac{N^{2}}{2^{\Theta(\sqrt{\log N})}}\right)=\Omega\left(\varepsilon^{2} \cdot \frac{n^{2}}{2^{\Theta(\sqrt{\log n})}}\right),
$$

as the number of vertices $n$ in the graph is $O(N)$. Setting $b=r / 4=\frac{n}{2^{\Theta(\sqrt{\log n})}}$ now concludes the proof of Theorem 2 (part $(i)$ of the theorem already follows from Observation 4.2).

\subsection{The Inverse Unique-Reach Problem}

In addition to the unique-reach problem, we also need another (almost identical) variant of this problem which we call the inverse of the unique-reach problem, denoted by $\overleftarrow{\text { unique-reach. This }}$ problem is basically what one would naturally expect if we reverse the direction of all edges in an instance of unique-reach and ask for finding the unique vertex that can now reach the end-vertex $t$ (corresponding to $s$ ). Formally, we define this problem as follows.

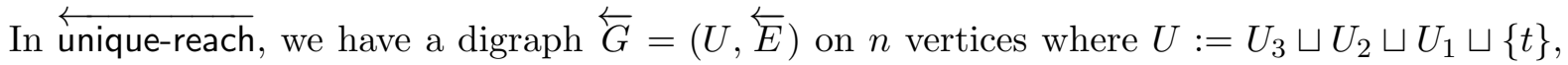
all edges of the graph are directed from $U_{1}$ to $t$ or some $U_{i+1}$ to $U_{i}$ for $i \in$ [2], and we are promised that there is a unique vertex $t^{\star}$ in $U_{3}$ that can reach $t$. The goal is to find this vertex $t^{\star}$, or rather, internal $\varepsilon$-solve it exactly as in Definition 4.1. As before, the edges between $U_{2}$ and $U_{1}$, 
denoted by $\overleftarrow{E}_{A}$, are given to Alice, and the remaining edges, denoted by $\overleftarrow{E}_{B}$, are given to Bob. The communication is also one-way from Alice to Bob.

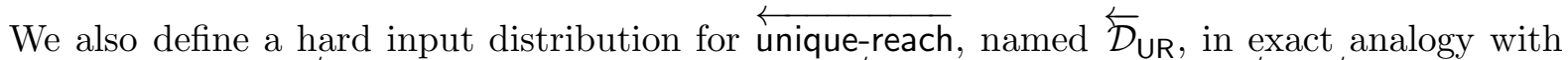
D्R for unique-reach: $\overleftarrow{\mathcal{D}}_{\mathrm{UR}}$ is a distribution over graphs $\overleftarrow{G}=\left(U_{3} \sqcup U_{2} \sqcup U_{1} \sqcup\{t\}, \overleftarrow{E}_{A} \sqcup \overleftarrow{E}_{B}\right)$, obtained by sampling a graph $G=\left(\{s\} \sqcup V_{1} \sqcup V_{2} \sqcup V_{3}, E_{A} \sqcup E_{B}\right)$ from $\mathcal{D}_{\mathrm{UR}}$, setting $U_{3}=V_{3}, U_{2}=V_{2}, U_{1}=V_{1}$, and $t=s$, and reversing the direction of all edges in $E_{A}$ and $E_{B}$ to obtain $\overleftarrow{E}_{A}$ and $\overleftarrow{E}_{B}$

\section{The st-Reachability Communication Problem}

We now define the main two-player communication problem (the setting of this problem is rather non-standard in terms of the communication model).

Problem 3 (st-reachability). Consider a digraph $G=(V, E)$ with two designated vertices $s, t$ and $E:=E_{1} \sqcup E_{2} \sqcup E_{3}$. The goal is to determine whether or not $s$ can reach $t$ in $G$.

Initially, Alice receives $E_{1}$ and $B o b$ receives $E_{2}$ (the vertices $s, t$ are known to both players). Next, Alice and Bob will have one round of communication by Alice sending a message $\Pi_{A 1}$ to Bob and Bob responding back with a message $\Pi_{B 1}$. At this point, the edges $E_{3}$ are revealed to both players. Finally, Alice is allowed to send yet another message $\Pi_{A 2}$ to Bob (which this time depends on $E_{3}$ as well) and Bob outputs the answer (again also a function of $E_{3}$ ).

The following theorem is the main result of our paper.

Theorem 3. For any $\varepsilon \in\left(n^{-1 / 2}, 1 / 2\right)$, any communication protocol for st-reachability that succeeds with probability at least $\frac{1}{2}+\varepsilon$ requires $\Omega\left(\varepsilon^{2} \cdot \frac{n^{2}}{2^{\Theta(\sqrt{\log n})}}\right)$ bits of communication.

We note that the $n^{-1 / 2}$ lower bound on $\varepsilon$ in Theorem 3 is not sacrosanct and any term which is $\omega\left(\frac{\log n}{b}\right)$ still works where $b=\frac{n}{2^{\Theta(\sqrt{\log n})}}$ is the parameter in Theorem 2 .

\subsection{A Hard Distribution for st-reachability}

Recall the distributions $\mathcal{D}_{\text {UR }}$ and $\overleftarrow{\mathcal{D}}_{\text {UR }}$ from Section 4 . We will use them to define our distribution for st-reachability. See Figure 2 for an illustration.

Distribution $\mathcal{D}_{\mathrm{ST}}$. A hard input distribution for the st-reachability problem.

(1) Let $V:=\{s\} \sqcup V_{1} \sqcup V_{2} \sqcup V_{3} \sqcup U_{3} \sqcup U_{2} \sqcup U_{1} \sqcup\{t\}$ - each $V_{i}$ or $U_{i}$ is called a layer of $G$ (this partitioning is known to both players).

(2) Sample the graph $G_{1}:=\left(V_{3} \sqcup U_{3}, E_{1}\right)$ by picking each edge $(v, u) \in V_{3} \times U_{3}$ independently and with probability half.

(3) Sample the following two graphs independently:

(i) $H:=\left(\{s\} \sqcup V_{1} \sqcup V_{2} \sqcup V_{3}, E_{A} \sqcup E_{B}\right)$ sampled from the distribution $\mathcal{D}_{\mathrm{UR}}$;

(ii) $\overleftarrow{H}:=\left(U_{3} \sqcup U_{2} \sqcup U_{1} \sqcup\{t\}, \overleftarrow{E}_{A} \sqcup \overleftarrow{E}_{B}\right)$ sampled from the distribution $\overleftarrow{\mathcal{D}}_{\text {UR }}$

(4) The initial input to Alice and Bob are, respectively, $E_{1}$ and $E_{2}:=E_{A} \cup \overleftarrow{E}_{A}$, and the input revealed to both players in the second round is $E_{3}:=E_{B} \cup \overleftarrow{E}_{B}$ 


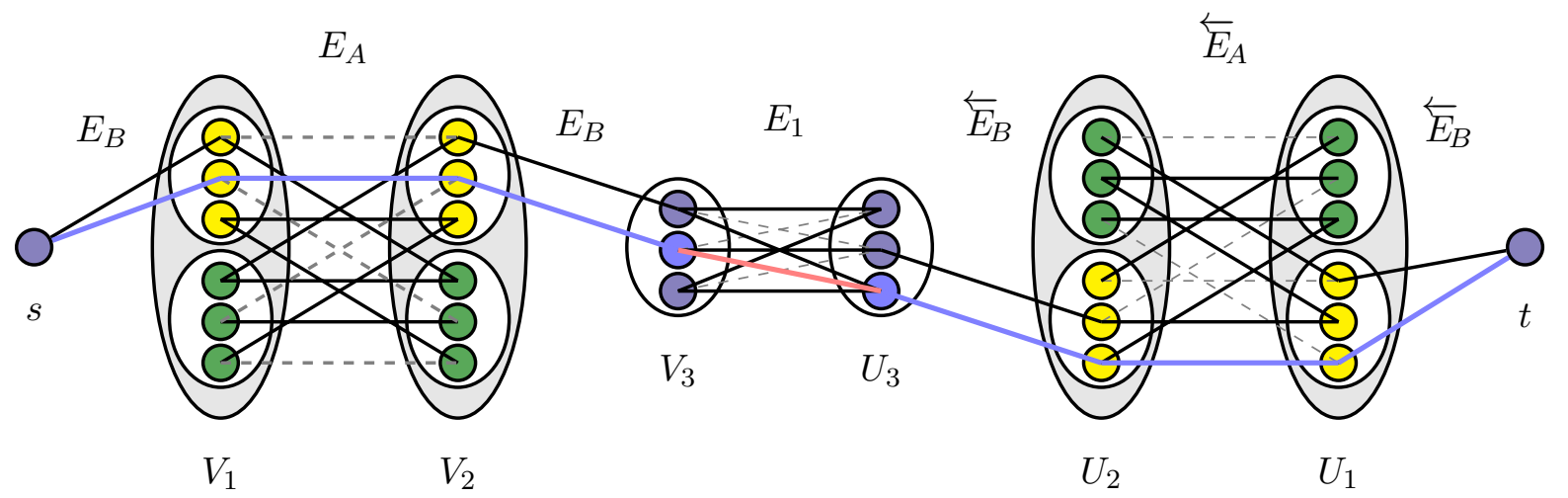

Figure 2: An illustration of the input distribution $\mathcal{D}_{\mathrm{ST}}$. Here, the directions of all edges are from left to right and hence omitted. The vertices $s^{\star} \in V_{3}$ and $t^{\star} \in U_{3}$ are marked blue and the potential edge $\left(s^{\star}, t^{\star}\right)$ is marked red-existence or non-existence of this edge uniquely determines whether or not $s$ can reach $t$ in $G$.

To avoid potential confusion, we should note right away that Bob in the distribution $\mathcal{D}_{\mathrm{ST}}$ is receiving the input of Alice in $\mathcal{D}_{\text {UR }}$ and $\overleftarrow{\mathcal{D}}_{\text {UR }}$

Observation 5.1. The following two remarks are in order:

(i) The distributions of $E_{1}, H$, and $\overleftarrow{H}$ are mutually independent in $\mathcal{D}_{\mathrm{ST}}$

(ii) $s$ can reach $t$ in $G$ iff the edge $\left(s^{\star}, t^{\star}\right) \in E_{1}$.

(proof: the only vertex in $V_{3}$ reachable from $s$ is $s^{\star}$ and the only vertex in $U_{3}$ that reaches $t$ is $t^{\star}$, thus the only potential $s$-t path is $s \rightsquigarrow s^{\star} \rightarrow t^{\star} \rightsquigarrow t$.)

\subsection{Setup and Notation}

Let $\pi_{\mathrm{ST}}$ be any deterministic protocol for st-reachability over the distribution $\mathcal{D}_{\mathrm{ST}}$ with

$$
\mathrm{CC}\left(\pi_{\mathrm{ST}}\right)=o\left(\varepsilon^{2} \cdot b^{2}\right),
$$

where $b:=\frac{n}{2^{\Theta(\sqrt{\log n})}}$ is the parameter in Theorem 2 for instances of $\mathcal{D}_{\mathrm{UR}}$ and $\overleftarrow{\mathcal{D}}_{\mathrm{UR}}$. We will prove that the probability that $\pi_{\mathrm{ST}}$ outputs the correct answer to st-reachability is $\frac{1}{2}+o(\varepsilon)$, hence proving Theorem 3 for deterministic protocols. The results for randomized protocols follows immediately from this and an averaging argument (i.e., the easy direction of Yao's minimax principle [87]).

To facilitate our proofs, the following notation would be useful. For brevity, we use

$$
\Pi:=\left(\Pi_{A 1}, \Pi_{B 1}, \Pi_{A 2}\right),
$$

(the messages communicated by Alice and Bob in both rounds of the protocol $\pi_{\mathrm{ST}}$ )

$$
Z_{1}:=\left(\Pi_{A 1}, \Pi_{B 1}, E_{3}\right),
$$

(the extra information known to Alice at the end of the first round of communication)

$$
Z_{2}:=\left(\Pi, E_{3}, s^{\star}, t^{\star}\right) \text {. }
$$

(the extra information known to Bob at the end of the second round of communication)

We also use $O \in\{0,1\}$ to denote the output Bob at the end of the protocol.

For any pair of vertices $v, u \in V_{3} \times U_{3}$, we use the notation $E_{1}(v, u) \in\{0,1\}$ to denote whether or not the edge $(v, u) \in E_{1}$. For a fixed choice of $E_{3}=E_{B} \cup \overleftarrow{E}_{B}$ in $\mathcal{D}_{\mathrm{ST}}$, we use $V_{3}^{\star}$ and $U_{3}^{\star}$ to 
denote the sets from which $s^{\star}$ and $t^{\star}$ are chosen uniformly at random from conditioned on $E_{B}$ and $\overleftarrow{E}_{B}$, respectively (see part $(i)$ of Theorem 2 ). We also define:

$$
E_{1}\left(V_{3}^{\star}, U_{3}^{\star}\right):=\left\{E_{1}\left(v_{i}, u_{i}\right) \mid\left(v_{i}, u_{i}\right) \in V_{3}^{\star} \times U_{3}^{\star}\right\} .
$$

We further assume a fixed arbitrary ordering of pairs $v, u \in V_{3} \times U_{3}$ and define:

$$
E_{1}^{<(v, u)}:=E_{1}\left(v_{1}, u_{1}\right), E_{1}\left(v_{2}, u_{2}\right), \ldots
$$

for all pairs $\left(v_{i}, u_{i}\right) \in E_{1}\left(V_{3}^{\star}, U_{3}^{\star}\right)$ that appear before $(v, u)$ in this ordering (note that we ignore the other edges of $E_{1}$ that are not in $E_{1}\left(V_{3}^{\star}, U_{3}^{\star}\right)$ here).

Throughout the proof, we will do a couple of reductions from our lower bound for unique-reach (on the distribution $\mathcal{D}_{\mathrm{ST}}$ ). To avoid ambiguity, in these cases, we use the notation "AlicesT and BobsT" to denote the players in the protocol $\pi_{\mathrm{ST}}$ for the st-reachability problem and use "Alice $\mathrm{UR}$ and Bobur" to denote the players in the protocol for the unique-reach problem (similar notation will be used for $\overleftarrow{\text { unique-reach }}$ as well). As an aside, we emphasize that in these reductions an "Alice-player" in one instance may need to play the role of a "Bob-player" in the other instance.

\section{Crucial Independence Properties}

The following independence properties are crucial for our proofs. They are all based on the rectangle property of communication protocols and part $(i)$ of Observation 5.1.

$$
\begin{aligned}
& \Pi_{A 2} \perp \mathrm{s}^{\star}, \mathrm{t}^{\star} \mid \mathrm{Z}_{1} \\
& \mathrm{E}_{1} \perp \mathrm{s}^{\star}, \mathrm{t}^{\star} \mid \mathrm{Z}_{1}, \Pi_{A 2} \\
& \mathrm{E}_{2} \perp \mathrm{E}_{1}\left(\mathrm{~s}^{\star}, \mathrm{t}^{\star}\right) \mid \mathrm{Z}_{1}, \mathrm{Z}_{2} .
\end{aligned}
$$

Proof of Eq (9).

$$
\begin{aligned}
\mathbb{I}\left(\mathrm{s}^{\star}, \mathrm{t}^{\star} ; \Pi_{A 2} \mid \mathrm{Z}_{1}\right) & \left.=\mathbb{I}\left(\mathrm{s}^{\star}, \mathrm{t}^{\star} ; \Pi_{A 2} \mid \Pi_{A 1}, \Pi_{A 2}, \mathrm{E}_{3}\right) \quad \text { (by the definition of } Z_{1}=\left(\Pi_{A 1}, \Pi_{B 1}, E_{3}\right)\right) \\
& \leqslant \mathbb{I}\left(\mathrm{E}_{2} ; \Pi_{A 2} \mid \Pi_{A 1}, \Pi_{B 1}, \mathrm{E}_{3}\right)
\end{aligned}
$$

(by the data processing inequality (Fact A.1-(5)) as $\mathrm{s}^{\star}, \mathrm{t}^{\star}$ is fixed by $\mathrm{E}_{2}$ conditioned on $\mathrm{E}_{3}$ )

$$
\leqslant \mathbb{I}\left(\mathrm{E}_{2} ; \mathrm{E}_{1} \mid \Pi_{A 1}, \Pi_{B 1}, \mathrm{E}_{3}\right)
$$

(by the data processing inequality (Fact A.1-(5)) as $\Pi_{A 2}$ is fixed by $\mathrm{E}_{1}$ conditioned on $\mathrm{E}_{3}, \Pi_{B 1}$ )

$$
\leqslant \mathbb{I}\left(\mathrm{E}_{2} ; \mathrm{E}_{1} \mid \Pi_{A 1}, \mathrm{E}_{3}\right)
$$

(by Proposition A.4 since $\Pi_{B 1} \perp \mathrm{E}_{1} \mid \mathrm{E}_{2}, \Pi_{A 1}, \mathrm{E}_{3}$ as $\mathrm{E}_{2}, \Pi_{A 1}$ fixes $\Pi_{B 1}$ )

$$
\leqslant \mathbb{I}\left(E_{2} ; E_{1} \mid E_{3}\right) \quad \text { (by Proposition A.4 since } \Pi_{A 1} \perp E_{2} \mid E_{1}, E_{3} \text { as } E_{1} \text { fixes } \Pi_{A 1} \text { ) }
$$$$
=0 \text {. } \quad \text { (by Fact A.1-(2) since } \mathrm{E}_{1} \perp \mathrm{E}_{2}, \mathrm{E}_{3} \text { by part (i) of Observation 5.1) }
$$

The proof now follows from Fact A.1-(2).

Proof of $E q(10)$.

$$
\mathbb{I}\left(\mathrm{s}^{\star}, \mathrm{t}^{\star} ; \mathrm{E}_{1} \mid \mathrm{Z}_{1}, \Pi_{A 2}\right) \leqslant \mathbb{I}\left(\mathrm{E}_{1} ; \mathrm{E}_{2} \mid \Pi_{A 1}, \Pi_{B 1}, \Pi_{A 2}, \mathrm{E}_{3}\right)
$$

(by the data processing inequality (Fact A.1-(5)) as $\mathrm{s}^{\star}, \mathrm{t}^{\star}$ is fixed by $\mathrm{E}_{2}$ conditioned on $\mathrm{E}_{3}$ )

$$
\leqslant \mathbb{I}\left(\mathrm{E}_{1} ; \mathrm{E}_{2} \mid \Pi_{A 1}, \Pi_{B 1}, \mathrm{E}_{3}\right)
$$

(by Proposition A.4 since $\Pi_{A 2} \perp \mathrm{E}_{2} \mid \mathrm{E}_{1}, \Pi_{A 1}, \Pi_{B 1}, \mathrm{E}_{3}$ as $\mathrm{E}_{1}, \Pi_{B 1}, \mathrm{E}_{3}$ fixes $\Pi_{A 2}$ )

$$
=0
$$

as was shown in the previous proof. The proof now follows from Fact A.1-(2). 
Proof of $E q(11)$.

$$
\begin{aligned}
\mathbb{I}\left(\mathrm{E}_{1}\left(\mathrm{~s}^{\star}, \mathrm{t}^{\star}\right) ; \mathrm{E}_{2} \mid \mathrm{Z}_{1}, \mathrm{Z}_{2}\right) & =\mathbb{I}\left(\mathrm{E}_{1}\left(\mathrm{~s}^{\star}, \mathrm{t}^{\star}\right) ; \mathrm{E}_{2} \mid \Pi_{A 1}, \Pi_{B 1}, \Pi_{A 2}, \mathrm{E}_{3}, \mathrm{~s}^{\star}, \mathrm{t}^{\star}\right) \\
& \leqslant \mathbb{I}\left(\mathrm{E}_{1} ; \mathrm{E}_{2} \mid \Pi_{A 1}, \Pi_{B 1}, \Pi_{A 2}, \mathrm{E}_{3}, \mathrm{~s}^{\star}, \mathrm{t}^{\star}\right)
\end{aligned}
$$

(by the data processing inequality (Fact A.1-(5)) as $\mathrm{E}_{1}\left(\mathrm{~s}^{\star}, \mathrm{t}^{\star}\right)$ is determined by $\mathrm{E}_{1} \mid \mathrm{s}^{\star}, \mathrm{t}^{\star}$ ) $\leqslant \mathbb{I}\left(\mathrm{E}_{1} ; \mathrm{E}_{2} \mid \Pi_{A 1}, \Pi_{B 1}, \Pi_{A 2}, \mathrm{E}_{3}\right)$

(by Proposition A.4 since $\mathrm{s}^{\star}, \mathrm{t}^{\star} \perp \mathrm{E}_{1} \mid \mathrm{E}_{2}, \Pi_{A 1}, \Pi_{B 1}, \Pi_{A 2}, \mathrm{E}_{3}$ as $\mathrm{E}_{2}, \mathrm{E}_{3}$ fixes $\mathrm{s}^{\star}, \mathrm{t}^{\star}$ ) $=0$,

as was shown in the previous proof. The proof now follows from Fact A.1-(2).

\subsection{Part One: The First Round of Communication}

In the following lemma, we prove that after the first round of the protocol, the (joint) distribution of $\left(s^{\star}, t^{\star}\right)$ conditioned on $Z_{1}=\left(\Pi_{A 1}, \Pi_{B 1}, E_{3}\right)$ is almost the same as if we only conditioned on $E_{3}$. This is basically through a reduction from Theorem 2 considering $s^{\star}, t^{\star}$ are distributed (originally) according to $\mathcal{D}_{\text {UR }}$ and $\overleftarrow{\mathcal{D}}_{\text {UR }}$ and the public information $E_{3}$ provides the input of Bob in the instances of unique-reach and $\overleftarrow{\text { unique-reach }}$ in this reduction.

Lemma 5.2. $\mathbb{E}_{\mathrm{Z}_{1}}\left\|\operatorname{dist}\left(\mathrm{s}^{\star}, \mathrm{t}^{\star} \mid Z_{1}\right)-\operatorname{dist}\left(\mathrm{s}^{\star}, \mathrm{t}^{\star} \mid E_{3}\right)\right\|_{\mathrm{tvd}}=o(\varepsilon)$.

We start with writing,

$$
\begin{aligned}
& \text { LHS in Lemma } 5.2=\underset{\mathrm{Z}_{1}}{\mathbb{E}}\left\|\operatorname{dist}\left(\mathrm{s}^{\star}, \mathrm{t}^{\star} \mid Z_{1}\right)-\operatorname{dist}\left(\mathrm{s}^{\star}, \mathrm{t}^{\star} \mid E_{3}\right)\right\|_{\mathrm{tvd}} \\
& \begin{aligned}
\leq \underset{\mathrm{Z}_{1}}{\mathbb{E}}\left\|\operatorname{dist}\left(\mathrm{s}^{\star} \mid Z_{1}\right)-\operatorname{dist}\left(\mathrm{s}^{\star} \mid E_{3}\right)\right\|_{\mathrm{tvd}} \\
\quad+\underset{\mathrm{Z}_{1}}{\mathbb{E}} \underset{\mathrm{s}^{\star} \mid Z_{1}}{\mathbb{E}}\left\|\operatorname{dist}\left(\mathrm{t}^{\star} \mid Z_{1}, \mathrm{~s}^{\star}\right)-\operatorname{dist}\left(\mathrm{t}^{\star} \mid E_{3}, \mathrm{~s}^{\star}\right)\right\|_{\mathrm{tvd}} \\
=\underset{\mathrm{Z}_{1}}{\mathbb{E}}\left\|\operatorname{dist}\left(\mathrm{s}^{\star} \mid Z_{1}\right)-\operatorname{dist}\left(\mathrm{s}^{\star} \mid E_{B}\right)\right\|_{\mathrm{tvd}} \\
\quad+\underset{\mathrm{Z}_{1}}{\mathbb{E}} \underset{\mathrm{s}^{\star} \mid Z_{1}}{\mathbb{E}}\left\|\operatorname{dist}\left(\mathrm{t}^{\star} \mid Z_{1}, \mathrm{~s}^{\star}\right)-\operatorname{dist}\left(\mathrm{t}^{\star} \mid \overleftarrow{E}_{B}\right)\right\|_{\mathrm{tvd}},
\end{aligned}
\end{aligned}
$$

where the final equality holds because $E_{3}=E_{B} \cup \overleftarrow{E}_{B}, \mathrm{~s}^{\star} \perp \overleftarrow{\mathrm{E}}_{B} \mid E_{B}$ and $\mathrm{t}^{\star} \perp \mathrm{s}^{\star}, \mathrm{E}_{B} \mid \overleftarrow{E}_{B}$ by Observation 5.1. In the following two claims, we bound each of the terms in Eq (12) by $o(\varepsilon)$.

Claim 5.3. $\mathbb{E}_{\mathrm{Z}_{1}}\left\|\operatorname{dist}\left(\mathrm{s}^{\star} \mid \mathrm{Z}_{1}\right)-\operatorname{dist}\left(\mathrm{s}^{\star} \mid E_{B}\right)\right\|_{\mathrm{tvd}}=o(\varepsilon)$.

Proof. Consider the following one-way protocol $\pi_{\mathrm{UR}}$ for instances $H:=\left(\{s\} \sqcup V_{1} \sqcup V_{2} \sqcup V_{3}, E_{A} \sqcup E_{B}\right)$ of unique-reach sampled from $\mathcal{D}_{\text {UR }}$ between the two players Alice UR and BobuR:

(i) Alice UR and BobUR sample $\overleftarrow{E}_{B} \sim \mathcal{D}_{\mathrm{ST}} \mid H$ using public randomness (this is doable without either player knowing $H$ by part (i) of Observation 5.1).

(ii) Alice UR samples $\overleftarrow{E}_{A} \sim \mathcal{D}_{\mathrm{ST}} \mid H, \overleftarrow{E}_{B}$ and $E_{1} \sim \mathcal{D}_{\mathrm{ST}} \mid H, \overleftarrow{E}_{A}, \overleftarrow{E}_{B}$ using private randomness (again, this is doable by part $(i)$ of Observation 5.1).

(iii) Alice UR has access to the first-round input of Alice $S$ T and BobsT and thus can generate $\Pi_{A 1}$ and $\Pi_{B 1}$ and send them to Bobur.

(iv) Bobur has access to $E_{3}$ and can compute $\operatorname{dist}\left(s^{\star} \mid \Pi_{A 1}, \Pi_{B 1}, E_{3}\right)$. 
Let $\delta \in(0,1)$ be the parameter for which $\pi_{\mathrm{UR}}$ internal $\delta$-solves unique-reach on $\mathcal{D}_{\mathrm{UR}}$. As such,

$$
\begin{aligned}
\delta & =\underset{\Pi_{\mathrm{UR}}, \mathrm{E}_{B}}{\mathbb{E}}\left\|\operatorname{dist}\left(\mathrm{s}^{\star} \mid \Pi_{\mathrm{UR}}, E_{B}\right)-\operatorname{dist}\left(\mathrm{s}^{\star} \mid E_{B}\right)\right\|_{\mathrm{tvd}} \\
& =\underset{\Pi_{A 1}, \Pi_{B 1}, \overleftarrow{\mathrm{E}}_{B}, \mathrm{E}_{B}}{\mathbb{E}}\left\|\operatorname{dist}\left(\mathrm{s}^{\star} \mid \Pi_{A 1}, \Pi_{B 1}, \overleftarrow{E}_{B}, E_{B}\right)-\operatorname{dist}\left(\mathrm{s}^{\star} \mid E_{B}\right)\right\|_{\mathrm{tvd}}
\end{aligned}
$$

(as the public randomness in $\pi_{\mathrm{UR}}$ is $\overleftarrow{E}_{B}$ and the message is $\Pi_{A 1}, \Pi_{B 1}$ )

$$
\begin{aligned}
& =\underset{\mathrm{Z}_{1}}{\mathbb{E}}\left\|\operatorname{dist}\left(\mathrm{s}^{\star} \mid Z_{1}\right)-\operatorname{dist}\left(\mathrm{s}^{\star} \mid E_{B}\right)\right\|_{\mathrm{tvd}} \quad\left(\text { as } Z_{1}=\left(\Pi_{A 1}, \Pi_{B 1}, E_{B}, \overleftarrow{E}_{B}\right)\right) \\
& =\text { LHS in Claim 5.3. }
\end{aligned}
$$

On the other hand, since

$$
\mathrm{CC}\left(\pi_{\mathrm{UR}}\right) \leqslant \mathrm{CC}\left(\pi_{\mathrm{ST}}\right)=o\left(\varepsilon^{2} \cdot b^{2}\right)=o\left(\varepsilon^{2} \cdot n \cdot b\right), \quad \quad(\text { by Eq }(8) \text { and since } b<n)
$$

by Theorem 2 , we should have that $\delta=o(\varepsilon)$, hence proving the claim.

Claim 5.4. $\mathbb{E}_{\mathrm{Z}_{1}} \mathbb{E}_{\mathrm{s}^{\star} \mid \mathrm{Z}_{1}}\left\|\operatorname{dist}\left(\mathrm{t}^{\star} \mid \mathrm{Z}_{1}, s^{\star}\right)-\operatorname{dist}\left(\mathrm{t}^{\star} \mid \overleftarrow{E}_{B}\right)\right\|_{\mathrm{tvd}}=o(\varepsilon)$

Proof. The proof of this claim is similar to that of Claim 5.3 with some minor (yet crucial) changes. Consider the following one-way protocol $\pi_{\mathrm{UR}}$ for instances $\overleftarrow{H}:=\left(U_{3} \sqcup U_{2} \sqcup U_{1} \sqcup\{t\}, \overleftarrow{E}_{A} \sqcup \overleftarrow{E}_{B}\right)$

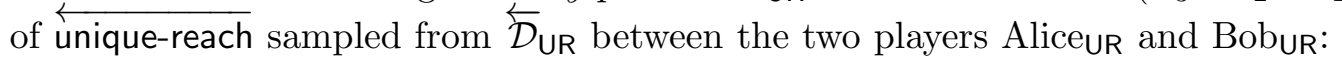

(i) Alice UR and BobUR sample $E_{B} \sim \mathcal{D}_{\mathrm{ST}} \mid \overleftarrow{H}$ using public randomness (this is doable without either player knowing $\overleftarrow{H}$ by part $(i)$ of Observation 5.1)

(ii) Alice $_{\mathrm{UR}}$ samples $E_{A} \sim \mathcal{D}_{\mathrm{ST}} \mid \overleftarrow{H}, E_{B}$ and $E_{1} \sim \mathcal{D}_{\mathrm{ST}} \mid \overleftarrow{H}, E_{A}, E_{B}$ using private randomness (again, this is doable by part $(i)$ of Observation 5.1).

(iii) Aliceur has access to the first-round input of Alicest and Bobst and thus can generate $\Pi_{A 1}$ and $\Pi_{B 1}$ and send them to Bobur. Additionally, Aliceur computes the vertex $s^{\star}$ and sends that also to Bobur (this is doable as Alicest has full information about $E_{A} \cup E_{B}$ ).

(iv) Bobur has access to $E_{3}$, and the vertex $s^{\star}$ and can thus compute $\operatorname{dist}\left(t^{\star} \mid \Pi_{A 1}, \Pi_{B 1}, E_{3}, s^{\star}\right)$.

Let $\delta \in(0,1)$ be the parameter for which $\pi_{\mathrm{UR}}$ internal $\delta$-solves $\overleftarrow{\text { unique-reach }}$ on $\overleftarrow{\mathcal{D}}_{\mathrm{UR}}$. As such,

$$
\begin{aligned}
\delta & =\underset{\Pi_{\mathrm{UR}}, \overleftarrow{\mathrm{E}}_{B}}{\mathbb{E}}\left\|\operatorname{dist}\left(\mathrm{t}^{\star} \mid \Pi_{\mathrm{UR}}, \overleftarrow{E}_{B}\right)-\operatorname{dist}\left(\mathrm{t}^{\star} \mid \overleftarrow{E}_{B}\right)\right\|_{\mathrm{tvd}} \quad \text { (by Definition 4.1) } \\
= & \underset{\Pi_{A 1}, \Pi_{B 1}, \mathrm{~s}^{\star}, \mathrm{E}_{B}, \overleftarrow{\mathrm{E}}_{B}}{\mathbb{E}}\left\|\operatorname{dist}\left(\mathrm{t}^{\star} \mid \Pi_{A 1}, \Pi_{B 1}, E_{B}, s^{\star}, \overleftarrow{E}_{B}\right)-\operatorname{dist}\left(\mathrm{t}^{\star} \mid \overleftarrow{E}_{B}\right)\right\|_{\mathrm{tvd}} \\
& \left.\quad \text { as the public randomness in } \pi_{\mathrm{UR}} \text { is } E_{B} \text { and the message is } \Pi_{A 1}, \Pi_{B 1}, s^{\star}\right) \\
= & \underset{\mathrm{Z}_{1}, \mathrm{~s}^{\star}}{\mathbb{E}}\left\|\operatorname{dist}\left(\mathrm{t}^{\star} \mid Z_{1}, s^{\star}\right)-\operatorname{dist}\left(\mathrm{t}^{\star} \mid \overleftarrow{E}_{B}\right)\right\|_{\mathrm{tvd}} \quad\left(\text { as } Z_{1}=\left(\Pi_{A 1}, \Pi_{B 1}, E_{B}, \overleftarrow{E}_{B}\right)\right) \\
= & \text { LHS in Claim 5.4. }
\end{aligned}
$$

On the other hand, since the vertex $s^{\star}$ communicated by AliceuR requires additional $O(\log n)$ bits on top of the message of $\pi_{\mathrm{ST}}$, we have,

$$
\mathrm{CC}\left(\pi_{\mathrm{UR}}\right) \leqslant \mathrm{CC}\left(\pi_{\mathrm{ST}}\right)+O(\log n)=o\left(\varepsilon^{2} \cdot b^{2}\right)+O(\log n)=o\left(\varepsilon^{2} \cdot n \cdot b\right) .
$$

(by Eq (8) and since $b<n$ and $\varepsilon>n^{-1 / 2}$ and so $\varepsilon^{2} \cdot n \cdot b \gg \log n$ )

By Theorem 2, this implies that $\delta=o(\varepsilon)$, hence proving the claim.

Lemma 5.2 now follows from plugging in the bounds in Claims 5.3 and 5.4 in Eq (12). 


\subsection{Part Two: The Second Round of Communication}

Lemma 5.2 implies that the extra information $Z_{1}$ available to Alice at the beginning of the second round does not change the distribution of $\left(s^{\star}, t^{\star}\right)$ by much. We use this to show that the message of Alice in the second round does not change the distribution of $E_{1}\left(s^{\star}, t^{\star}\right) \in\{0,1\}$ by much.

Lemma 5.5. $\mathbb{E}_{\mathrm{Z}_{1}, \mathrm{Z}_{2}}\left\|\operatorname{dist}\left(\mathrm{E}_{1}\left(s^{\star}, t^{\star}\right) \mid Z_{1}, Z_{2}\right)-\operatorname{dist}\left(\mathrm{E}_{1}\left(s^{\star}, t^{\star}\right)\right)\right\|_{\mathrm{tvd}}=o(\varepsilon)$.

As $Z_{2}=\left(\Pi, E_{3}, s^{\star}, t^{\star}\right), \Pi=\left(\Pi_{A 1}, \Pi_{B 1}, \Pi_{A 2}\right)$, and $E_{3}, \Pi_{A 1}, \Pi_{B 1}$ are also part of $Z_{1}$, we have,

$$
\begin{aligned}
\text { LHS in Lemma } 5.5 & =\underset{\mathrm{Z}_{1}, \Pi_{A 2}, \mathrm{~s}^{\star}, \mathrm{t}^{\star}}{\mathbb{E}}\left\|\operatorname{dist}\left(\mathrm{E}_{1}\left(s^{\star}, t^{\star}\right) \mid Z_{1}, \Pi_{A 2}, s^{\star}, t^{\star}\right)-\operatorname{dist}\left(\mathrm{E}_{1}\left(s^{\star}, t^{\star}\right)\right)\right\|_{\mathrm{tvd}} \\
& =\underset{\mathrm{Z}_{1}, \Pi_{A 2}, \mathrm{~s}^{\star}, \mathrm{t}^{\star}}{\mathbb{E}}\left\|\operatorname{dist}\left(\mathrm{E}_{1}\left(s^{\star}, t^{\star}\right) \mid Z_{1}, \Pi_{A 2}\right)-\operatorname{dist}\left(\mathrm{E}_{1}\left(s^{\star}, t^{\star}\right)\right)\right\|_{\mathrm{tvd}} \\
& \quad\left(\operatorname{as} \mathrm{E}_{1}\left(s^{\star}, t^{\star}\right) \perp \mathrm{s}^{\star}=s^{\star}, \mathrm{t}^{\star}=t^{\star} \mid Z_{1}, \Pi_{A 2}\right. \text { by Eq (10)) } \\
& \underset{\mathrm{z}_{1}, \Pi_{A 2} \mathrm{~s}^{\star}, \mathrm{t}^{\star} \mid Z_{1}, \Pi_{A 2}}{\mathbb{E}}\left\|\operatorname{dist}\left(\mathrm{E}_{1}\left(s^{\star}, t^{\star}\right) \mid Z_{1}, \Pi_{A 2}\right)-\operatorname{dist}\left(\mathrm{E}_{1}\left(s^{\star}, t^{\star}\right)\right)\right\|_{\mathrm{tvd}} \cdot \quad
\end{aligned}
$$

Intuitively, by Lemma 5.2 and $\mathrm{Eq}(9)$, we know that $\left(\mathrm{s}^{\star}, \mathrm{t}^{\star} \mid Z_{1}, \Pi_{A 2}\right)$ is distributed almost uniformly over a set determined only by $E_{3}$ (on average over $Z_{1}, \Pi_{A 2}$ ). Thus, in the following lemma, we first check what happens to Eq (13) if we switch this distribution to a truly uniform one, and then use this to conclude the proof.

Lemma 5.6. Suppose $(\mathrm{x}, \mathrm{y})$ is distributed as $\left(\mathrm{s}^{\star}, \mathrm{t}^{\star}\right) \mid \mathrm{E}_{3}$, i.e., uniformly at random over $V_{3}^{\star} \times U_{3}^{\star}$ for any $E_{3}$ (independent of the remaining variables). Then,

$$
\underset{Z_{1}, \Pi_{A 2}}{\mathbb{E}} \underset{\times, y \mid E_{3}}{\mathbb{E}}\left\|\operatorname{dist}\left(\mathrm{E}_{1}(x, y) \mid Z_{1}, \Pi_{A 2}\right)-\operatorname{dist}\left(\mathrm{E}_{1}(x, y)\right)\right\|_{\mathrm{tvd}}=o(\varepsilon)
$$

Proof. We first have,

LHS in Lemma $5.6=\underset{\mathrm{Z}_{1}, \Pi_{A 2}}{\mathbb{E}} \underset{\times, y \mid E_{3}}{\mathbb{E}}\left\|\operatorname{dist}\left(\mathrm{E}_{1}(x, y) \mid Z_{1}, \Pi_{A 2}, x, y\right)-\operatorname{dist}\left(\mathrm{E}_{1}(x, y)\right)\right\|_{\text {tvd }}$

(as $\mathrm{E}_{1}(x, y) \perp \mathrm{x}=x, \mathrm{y}=y \mid Z_{1}, \Pi_{A 2}$ by the definition of $\mathrm{x}, \mathrm{y}$ )

$$
\leqslant \underset{\mathrm{Z}_{1}, \Pi_{A 2}}{\mathbb{E}} \underset{x, y \mid E_{3}}{\mathbb{E}}\left[\sqrt{\frac{1}{2} \cdot \mathbb{D}\left(\operatorname{dist}\left(\mathrm{E}_{1}(x, y) \mid Z_{1}, \Pi_{A 2}, x, y\right) \| \operatorname{dist}\left(\mathrm{E}_{1}(x, y)\right)\right)}\right]
$$

(by Pinsker's inequality (Fact A.7))

$$
\leqslant \sqrt{\frac{1}{2} \cdot \underset{\mathrm{Z}_{1}, \Pi_{A 2}, \mathrm{x}, \mathrm{y}}{\mathbb{E}}\left[\mathbb{D}\left(\operatorname{dist}\left(\mathrm{E}_{1}(x, y) \mid Z_{1}, \Pi_{A 2}, x, y\right) \| \operatorname{dist}\left(\mathrm{E}_{1}(x, y)\right)\right)\right]}
$$

(by concavity of $\sqrt{\cdot}$ and Jensen's inequality)

$$
=\sqrt{\frac{1}{2} \cdot \mathbb{I}\left(\mathrm{E}_{1}(\mathrm{x}, \mathrm{y}) ; \mathrm{Z}_{1}, \Pi_{A 2}, \mathrm{x}, \mathrm{y}\right)} .
$$

We now bound the mutual information term in the RHS above. Recall that $\Pi=\left(\Pi_{A 1}, \Pi_{B 1}, \Pi_{A 2}\right)$ and $Z_{1}=\left(\Pi_{A 1}, \Pi_{B 1}, E_{3}\right)$. We have,

$$
\begin{aligned}
\mathbb{I}\left(E_{1}(x, y) ; Z_{1}, \Pi_{A 2}, x, y\right) & =\mathbb{I}\left(E_{1}(x, y) ; \Pi, E_{3}, x, y\right) \\
& =\mathbb{I}\left(E_{1}(x, y) ; E_{3}, x, y\right)+\mathbb{I}\left(E_{1}(x, y) ; \Pi \mid E_{3}, x, y\right) \\
& \quad \quad \text { by the chain rule of mutual information (Fact A.1-(4))) } \\
& \mathbb{I}\left(E_{1}(x, y) ; \Pi \mid E_{3}, x, y\right)
\end{aligned}
$$

(as the first term above is zero by Fact A.1-(2) since $E_{1}(x, y) \perp E_{3}, x, y$ by Observation 5.1) 


$$
\begin{aligned}
& =\underset{\mathrm{E}_{3}}{\mathbb{E}}\left[\sum_{(x, y) \in V_{3}^{\star} \times U_{3}^{\star}} \frac{1}{\left|V_{3}^{\star} \times U_{3}^{\star}\right|} \cdot \mathbb{I}\left(\mathrm{E}_{1}(x, y) ; \Pi \mid E_{3}, x, y\right)\right] \\
& \text { (as } \left.\operatorname{dist}\left(\mathrm{x}, \mathrm{y} \mid E_{3}\right) \text { is uniform over } V_{3}^{\star} \times U_{3}^{\star}\right) \\
& =\frac{1}{b^{2}} \cdot \underset{\mathrm{E}_{3}}{\mathbb{E}}\left[\sum_{(x, y) \in V_{3}^{\star} \times U_{3}^{\star}} \mathbb{I}\left(\mathrm{E}_{1}(x, y) ; \Pi \mid E_{3}, x, y\right)\right] \\
& \text { (by part }(i) \text { of Theorem 2, size of } V_{3}^{\star} \text { and } U_{3}^{\star} \text { is } b \text { ) } \\
& =\frac{1}{b^{2}} \cdot \underset{\mathrm{E}_{3}}{\mathbb{E}}\left[\sum_{(x, y) \in V_{3}^{\star} \times U_{3}^{\star}} \mathbb{I}\left(\mathrm{E}_{1}(x, y) ; \Pi \mid E_{3}\right)\right] \quad\left(\text { as } \mathrm{x}, \mathrm{y} \perp \mathrm{E}_{1}(x, y), \Pi \mid E_{3}\right) \\
& \leqslant \frac{1}{b^{2}} \cdot \underset{\mathrm{E}_{3}}{\mathbb{E}}\left[\sum_{(x, y) \in V_{3}^{\star} \times U_{3}^{\star}} \mathbb{I}\left(\mathrm{E}_{1}(x, y) ; \Pi \mid \mathrm{E}_{1}^{<(x, y)}, E_{3}\right)\right] \\
& \text { (by Proposition A.3 as } \mathrm{E}_{1}(x, y) \perp \mathrm{E}_{1}^{<(x, y)} \mid E_{3} \text { ) } \\
& =\frac{1}{b^{2}} \cdot \underset{\mathrm{E}_{3}}{\mathbb{E}}\left[\mathbb{I}\left(\mathrm{E}_{1}\left(V_{3}^{\star}, U_{3}^{\star}\right) ; \Pi \mid E_{3}\right)\right] \\
& \text { (by the chain rule of mutual information (Fact A.1-(4))) } \\
& \leqslant \frac{1}{b^{2}} \cdot \log |\operatorname{supp}(\Pi)| \\
& =\frac{1}{b^{2}} \cdot \mathrm{CC}\left(\pi_{\mathrm{ST}}\right) \text {. }
\end{aligned}
$$

(as $\pi_{\mathrm{ST}}$ is a deterministic protocol and thus $\Pi$ only contains its message) Since CC $\left(\pi_{\mathrm{ST}}\right)=o\left(\varepsilon^{2} \cdot b^{2}\right)$ by our assumption in Eq (8), we obtain the proof of the lemma.

We can now conclude the proof of Lemma 5.5. For any choice of $E_{3}$, define $\mathrm{x}, \mathrm{y}$ as random variables distributed as $\left(\mathrm{s}^{\star}, \mathrm{t}^{\star}\right) \mid E_{3}$ (as in the statement of Lemma 5.6). We have,

$$
\begin{aligned}
& \text { LHS in Lemma } 5.5=\underset{\mathrm{z}_{1}, \Pi_{A 2}}{\mathbb{E}} \underset{\mathrm{s}^{\star}, \mathrm{t}^{\star} \mid Z_{1}, \Pi_{A 2}}{\mathbb{E}}\left\|\operatorname{dist}\left(\mathrm{E}_{1}\left(s^{\star}, t^{\star}\right) \mid Z_{1}, \Pi_{A 2}\right)-\operatorname{dist}\left(\mathrm{E}_{1}\left(s^{\star}, t^{\star}\right)\right)\right\|_{\mathrm{tvd}} \\
& \leqslant \underset{\mathrm{z}_{1}, \Pi_{A 2}}{\mathbb{E}} \underset{\mathrm{x}, \mathrm{y} \mid Z_{1}, \Pi_{A 2}}{\mathbb{E}}\left\|\operatorname{dist}\left(\mathrm{E}_{1}(x, y) \mid Z_{1}, \Pi_{A 2}\right)-\operatorname{dist}\left(\mathrm{E}_{1}(x, y)\right)\right\|_{\mathrm{tvd}} \\
& \quad+\underset{\mathrm{z}_{1}, \Pi_{A 2}}{\mathbb{E}}\left\|\operatorname{dist}\left(\mathrm{s}^{\star}, \mathrm{t}^{\star} \mid Z_{1}, \Pi_{A 2}\right)-\operatorname{dist}\left(\mathrm{x}, \mathrm{y} \mid Z_{1}, \Pi_{A 2}\right)\right\|_{\mathrm{tvd}} \\
&=\underset{\mathrm{z}_{1}, \Pi_{A 2}}{\mathbb{E}} \underset{\mathrm{x}, \mathrm{y} \mid E_{3}}{\mathbb{E}}\left\|\operatorname{dist}\left(\mathrm{E}_{1}(x, y) \mid Z_{1}, \Pi_{A 2}\right)-\operatorname{dist}\left(\mathrm{E}_{1}(x, y)\right)\right\|_{\mathrm{tvd}} \quad \text { (by Fact A.6) } \\
& \quad+\underset{\mathrm{z}_{1}, \Pi_{A 2}}{\mathbb{E}}\left\|\operatorname{dist}\left(\mathrm{s}^{\star}, \mathrm{t}^{\star} \mid Z_{1}, \Pi_{A 2}\right)-\operatorname{dist}\left(\mathrm{x}, \mathrm{y} \mid E_{3}\right)\right\|_{\mathrm{tvd}} \quad\left(\text { as } \mathrm{x}, \mathrm{y} \perp \Pi \mid E_{3}\right) \\
&=o(\varepsilon)+\underset{\mathrm{z}_{1}, \Pi_{A 2}}{\mathbb{E}}\left\|\operatorname{dist}\left(\mathrm{s}^{\star}, \mathrm{t}^{\star} \mid Z_{1}, \Pi_{A 2}\right)-\operatorname{dist}\left(\mathrm{s}^{\star}, \mathrm{t}^{\star} \mid E_{3}\right)\right\|_{\mathrm{tvd}}
\end{aligned}
$$

(by Lemma 5.6 for the first term, and since $(\mathrm{x}, \mathrm{y}) \mid E_{3}$ is distributed the same as $\left(\mathrm{s}^{\star}, \mathrm{t}^{\star}\right) \mid E_{3}$ )

$$
\begin{aligned}
& =o(\varepsilon)+\underset{Z_{1}}{\mathbb{E}}\left\|\operatorname{dist}\left(\mathrm{s}^{\star}, \mathrm{t}^{\star} \mid Z_{1}\right)-\operatorname{dist}\left(\mathrm{s}^{\star}, \mathrm{t}^{\star} \mid E_{3}\right)\right\|_{\mathrm{tvd}} \\
& =o(\varepsilon) \text {. } \\
& \left(\text { as s }^{\star}, \mathrm{t}^{\star} \perp \Pi_{A 2}=\Pi_{A 2} \mid Z_{1} \text { by Eq }(9)\right)
\end{aligned}
$$

This concludes the proof of Lemma 5.5. 
Remark 5.7. This is a good place to state the reason we work with the notion of internal $\varepsilon$-solving instead of external $\varepsilon$-solving in [6]. Roughly speaking, we would like the distribution of $\left(s^{\star}, t^{\star}\right)$ to look "almost random" to Alice at the beginning of the second round, so that her second-round message does not reveal much information about $E_{1}\left(s^{\star}, t^{\star}\right)$. But this has to hold despite the fact that she has the entire set $E_{3}$ at the beginning of the second round (and is thus "internal" to the underlying communication problem for unique-reach and its inner set-intersection instance).

This issue was handled differently in [6] by working with four players instead of two in a way that the players were no longer internal to the problem they needed to solve. However, such a fix does not work for our purpose: even if we did not provide $E_{3}$ directly to Alice, Bob could have sent it to Alice using $O(n)$ communication which is negligible, and thus making Alice again internal to the communication problem (in the setting of [6], such an approach would require $\Omega\left(n^{2}\right)$ communication which is no longer negligible).

\subsection{Concluding the Proof of Theorem 3}

We are now ready to conclude the proof of Theorem 3. Lemma 5.5 implies that conditioning on $Z_{1}, Z_{2}$ does not change the distribution of $E_{1}\left(s^{\star}, t^{\star}\right)$ by much. By the independence property of Eq (11), we know that this continues to hold even if we further condition on the input of Bob, i.e., $E_{2}$. We use this to prove that the probability that $\pi_{\mathrm{ST}}$ outputs the correct answer is almost the same as random guessing.

Claim 5.8. $\operatorname{Pr}\left(\pi_{\mathrm{ST}}\right.$ outputs the correct answer $)=\frac{1}{2}+o(\varepsilon)$.

Proof. Recall that $O \in\{0,1\}$ is the output of the protocol by Bob which is a function of $E_{2}$ and $Z_{1}, Z_{2}$, namely, the input to Bob and the information revealed to him (either through $E_{3}$ or the protocol). Recall that by part (ii) of Observation $5.1, E_{1}\left(s^{\star}, t^{\star}\right)$ determines the correct answer. Let $\mathcal{B}(1 / 2)$ denote the Bernoulli distribution with mean $1 / 2$. We have,

$\operatorname{Pr}\left(\pi_{\mathrm{ST}}\right.$ outputs correctly $)=\underset{\mathrm{Z}_{1}, \mathrm{Z}_{2}, \mathrm{E}_{2}}{\mathbb{E}} \underset{x \sim \mathrm{E}_{1}\left(s^{\star}, t^{\star}\right) \mid Z_{1}, Z_{2}, E_{2}}{\operatorname{Pr}}(O=x)$

(note that conditioning on $\mathrm{Z}_{1}, \mathrm{Z}_{2}, \mathrm{E}_{2}$ fixes $\mathrm{O}=O$ but not $\mathrm{E}_{1}\left(s^{\star}, t^{\star}\right)$ )

$\leqslant \underset{\mathrm{Z}_{1}, \mathrm{Z}_{2}, \mathrm{E}_{2}}{\mathbb{E}}\left[\underset{x \sim \mathcal{B}(1 / 2)}{\operatorname{Pr}}(O=x)+\left\|\operatorname{dist}\left(\mathrm{E}_{1}\left(s^{\star}, t^{\star}\right) \mid Z_{1}, Z_{2}, E_{2}\right)-\mathcal{B}(1 / 2)\right\|_{\text {tvd }}\right]$

(by Fact A.6)

$\leqslant \frac{1}{2}+\underset{\mathrm{Z}_{1}, \mathrm{Z}_{2}, \mathrm{E}_{2}}{\mathbb{E}}\left\|\operatorname{dist}\left(\mathrm{E}_{1}\left(s^{\star}, t^{\star}\right) \mid Z_{1}, Z_{2}, E_{2}\right)-\mathcal{B}(1 / 2)\right\|_{\mathrm{tvd}}$

(as $O$ is fixed and $x$ is chosen uniformly at random from $\{0,1\}$ )

$=\frac{1}{2}+\underset{\mathrm{Z}_{1}, \mathrm{Z}_{2}}{\mathbb{E}}\left\|\operatorname{dist}\left(\mathrm{E}_{1}\left(s^{\star}, t^{\star}\right) \mid Z_{1}, Z_{2}\right)-\mathcal{B}(1 / 2)\right\|_{\mathrm{tvd}}$

(as $\mathrm{E}_{1}\left(s^{\star}, t^{\star}\right) \perp \mathrm{E}_{2}=E_{2} \mid Z_{1}, Z_{2}$ by $\mathrm{Eq}(11)$ )

$=\frac{1}{2}+\underset{\mathrm{Z}_{1}, \mathrm{Z}_{2}}{\mathbb{E}}\left\|\operatorname{dist}\left(\mathrm{E}_{1}\left(s^{\star}, t^{\star}\right) \mid Z_{1}, Z_{2}\right)-\operatorname{dist}\left(\mathrm{E}_{1}\left(s^{\star}, t^{\star}\right)\right)\right\|_{\mathrm{tvd}}$

(by definition of $E_{1}$ )

$=\frac{1}{2}+o(\varepsilon)$.

(by Lemma 5.5)

This finalizes the proof.

To conclude, we have shown that for any deterministic protocol $\pi_{\mathrm{ST}}$ with $\mathrm{CC}\left(\pi_{\mathrm{ST}}\right)=o\left(\varepsilon^{2} \cdot b^{2}\right)$, the probability that $\pi_{\mathrm{ST}}$ outputs the correct answer over the distribution $\mathcal{D}_{\mathrm{ST}}$ is only $\frac{1}{2}+o(\varepsilon)$. This 
can be extended directly to randomized protocols as by an averaging argument, we can always fix the randomness of any randomized protocol $\pi_{\mathrm{ST}}$ on the distribution $\mathcal{D}_{\mathrm{ST}}$ to obtain a deterministic protocol with the same error guarantee. Noting that $b=\frac{n}{2^{\Theta(\sqrt{\log n})}}$ concludes the proof of Theorem 3.

\section{Graph Streaming Lower Bounds}

We now obtain our graph streaming lower bounds by reductions from the st-reachability communication problem defined in Section 5. The first step of all these reductions is to show that one can simulate any two-pass graph streaming algorithm on graphs $G=(V, E)$ using a protocol in the setting of the st-reachability problem. The proof is via a standard simulation and is only provided for completeness considering the setting of st-reachability is rather non-standard.

Proposition 6.1. Any two-pass $S$-space streaming algorithm $\mathcal{A}$ on graphs $G=\left(V, E_{1} \sqcup E_{2} \sqcup E_{3}\right)$ of st-reachability can be simulated exactly by a communication protocol $\pi_{\mathcal{A}}$ with $\mathrm{CC}\left(\pi_{\mathcal{A}}\right)=O(S)$ and the communication-pattern restrictions of the st-reachability problem.

Proof. Given $G=\left(V, E_{1} \sqcup E_{2} \sqcup E_{3}\right)$ of st-reachability, we define the stream $\sigma=E_{1} \circ E_{2} \circ E_{3}$. We use $M_{i, j}$ for $i \in\{1,2\}$ and $j \in\{1,2,3\}$ as the memory content of $\mathcal{A}$ after the $i$-th pass over the set of edges $E_{j}$ in the stream $\sigma$. We note that since $\mathcal{A}$ is a streaming algorithm, $M_{i, j}$ is only a function of $M_{i, j-1}$ and $E_{j}$ with the exception that $M_{2,1}$ is a function of $M_{1,3}$ and $E_{1}$. The answer is also uniquely determined by $M_{2,3}$.

We now give the protocol $\pi_{\mathcal{A}}$ that allows Alice and Bob to simulate two passes of $\mathcal{A}$ on $\sigma$ :

(i) Given $E_{1}$, Alice runs $\mathcal{A}$ on $E_{1}$ and sends $M_{1,1}$ as the message $\Pi_{A 1}$.

(ii) Given $\Pi_{A 1}=M_{1,1}$ and $E_{2}$, Bob continues running $\mathcal{A}$ on $E_{2}$ and sends $M_{1,2}$ to Alice as $\Pi_{B 1}$. This finishes the first round of communication in $\pi_{\mathcal{A}}$ (but not the first pass of $\mathcal{A}$ yet).

(iii) Given $\Pi_{B 1}=M_{1,2}$ and $E_{3}$ at the beginning of the second round, Alice continues running $\mathcal{A}$ on $E_{3}$ which concludes the first pass of $\mathcal{A}$. Considering Alice also has $E_{1}$, she then continues the second pass by running $\mathcal{A}$ on $E_{1}$ for the second time and sending $M_{2,1}$ as $\Pi_{A 2}$ to Bob.

(iv) Given $\Pi_{A 2}=M_{2,1}$ and $E_{3}$ now and having $E_{2}$ from before, Bob can compute both $M_{2,2}$ and $M_{2,3}$ and outputs the same answer as $\mathcal{A}$.

It is straightforward to verify that communication cost of this protocol is $O(S)$ and it outputs the same exact answer as $\mathcal{A}$.

\subsection{Directed Reachability}

We obtain the following theorem for the directed reachability problem.

Theorem 4 (Formalization of Result 1). Any streaming algorithm that makes two passes over the edges of any n-vertex directed graph $G=(V, E)$ with two designated vertices $s, t \in V$ and outputs whether or not $s$ can reach $t$ in $G$ with probability at least $2 / 3$ requires $\Omega\left(\frac{n^{2}}{2^{\Theta(\sqrt{\log n})}}\right)$ space.

Theorem 4 follows immediately from Proposition 6.1 and our lower bound in Theorem 3 .

We also present some standard extension of this lower bound to other problems related to the directed reachability problem. 
- Estimating number of vertices reachable from a source: Consider any instance of the problem in Theorem 4 and connect $t$ to $2 n$ new vertices. In the new graph, if $s$ can reach $t$, then it can also reach at least $2 n$ other vertices, while if $s$ does not reach $t$, it can reach at most $n$ other vertices. Hence, the lower bound in Theorem 4 extends to this problem as well which was studied (in a similar format) in [60].

- Testing if $G$ is acyclic or not: Recall that the hard distribution of graphs in Theorem 3 and hence Theorem 4 is supported on acyclic graphs. If in these graphs, we connect $t$ to $s$ directly, then the graph remains acyclic iff $s$ cannot reach $t$. Hence, the lower bound in Theorem 4 extends to this problem as well.

- Approximating minimum feedback arc set: The lower bound for acyclicity implies the same bounds for any (multiplicative) approximation algorithm of minimum feedback arc set (the minimum number of edges to be deleted to make a graph acyclic) studied in [29].

\subsection{Bipartite Perfect Matching}

We obtain the following theorem for the bipartite perfect matching problem.

Theorem 5 (Formalization of Result 2). Any streaming algorithm that makes two passes over the edges of any $n$-vertex undirected bipartite graph $G=(L \sqcup R, E)$ and outputs whether or not $G$ has a perfect matching with probability at least $2 / 3$ requires $\Omega\left(\frac{n^{2}}{2^{\Theta(\sqrt{\log n})}}\right)$ space.

Proof. The proof is via a standard reduction from Theorem 4. Basically, we show that any algorithm for perfect matching problem can also be used to solve the $s$ - $t$ reachability problem within the same asymptotic space. We note that this reduction is folklore and we claim no novelty in this part.

Consider a directed graph $H=\left(V_{H}, E_{H}\right)$ and two vertices $s, t \in V_{H}$. Create the following bipartite graph $G=(L \sqcup R, E)$ :

- For any vertex $v \in V_{H} \backslash\{s, t\}$, there are two vertices $v^{\ell} \in L$ and $v^{r} \in R$. We also have a new vertex $s^{\ell} \in L$ and $t^{r} \in R$ corresponding to $s$ and $t$.

- For any (directed) edge $(u, v) \in E_{H}$, there is an (undirected) edge between $u^{\ell}$ and $v^{r}$ in $G$ (we assume without loss of generality that $s$ has no incoming edges and $t$ has no outgoing ones). Moreover, for any $v \in V_{H} \backslash\{s, t\}$, there is an (undirected) edge between $v^{\ell}$ and $v^{r}$.

We now claim that $s$ can reach $t$ in $H$ iff $G$ has a perfect matching. To see this, first consider the matching $M=\left\{\left(v^{\ell}, v^{r}\right) \mid v \in V_{H} \backslash\{s, t\}\right\}$ in $G$. In this matching, the only unmatched vertices are $s^{\ell}$ and $t^{r}$. Now note that any augmenting path of this matching $M$ in $G$ between $s^{\ell}$ and $t^{r}$ corresponds to a directed path from $s$ to $t$ in $H$. This implies that the only way for $G$ to have a perfect matching is if there is a $s$ - $t$ path in $H$ and vice versa.

As this reduction can be done "on the fly" in the streaming setting, the lower bound in theorem 5 follows from Theorem 4 immediately.

\subsection{Single-Source Shortest Path}

Finally, we have the following theorem for the shortest path problem. 
Theorem 6 (Formalization of Result 3). Any streaming algorithm that makes two passes over the edges of any $n$-vertex undirected graph $G=(V, E)$ with two designated vertices $s, t \in V$ and outputs the length of the shortest $s$ - $t$ path in $G$ with probability at least $2 / 3$ requires $\Omega\left(\frac{n^{2}}{2^{\Theta(\sqrt{\log n})}}\right)$ space.

The lower bound continues to hold even if the algorithm is allowed to output an estimate which, with probability at least $2 / 3$, is as large as the length of the shortest $s$-t path and strictly smaller than $9 / 7$ times the length of the shortest $s$ - $t$ path.

Proof. The proof is via a reduction from Theorem 3 and applying Proposition 6.1. Consider any graph $H$ in the support of the distribution $\mathcal{D}_{\mathrm{ST}}$ in Theorem 3 and assume we simply make all edges undirected to obtain a graph $G$. We claim that the length of the shortest $s$ - $t$ path in $G$ is 7 if $s$ can reach $t$ in $H$, and is otherwise at least 9 .

The proof is as follows: $(i)$ when $s$ can reach $t$ in $H$, we can simply take that path, which is of length 7 to get from $s$ to $t$ in $G$; (ii) in other case, we need to take at least one more back-edge (i.e., in $G$ go in the opposite direction of a directed edge in $H$ ) and one more forward edge to reach $t$ in $G$, making the path to be of length at least 9. See Figure 2 for an illustration.

The lower bound now follows from Theorem 3 and Proposition 6.1.

\section{References}

[1] K. J. Ahn and S. Guha. Graph sparsification in the semi-streaming model. In Automata, Languages and Programming, 36th Internatilonal Colloquium, ICALP 2009, Rhodes, Greece, July 5-12, 2009, Proceedings, Part II, pages 328-338, 2009. 1

[2] K. J. Ahn, S. Guha, and A. McGregor. Graph sketches: sparsification, spanners, and subgraphs. In Proceedings of the 31st ACM SIGMOD-SIGACT-SIGART Symposium on Principles of Database Systems, PODS 2012, Scottsdale, AZ, USA, May 20-24, 2012, pages 5-14, 2012. 1

[3] K. J. Ahn, S. Guha, and A. McGregor. Spectral sparsification in dynamic graph streams. In Approximation, Randomization, and Combinatorial Optimization. Algorithms and Techniques - 16th International Workshop, APPROX 2013, and 17th International Workshop, RANDOM 2013, Berkeley, CA, USA, August 21-23, 2013. Proceedings, pages 1-10, 2013. 1

[4] N. Alon, A. Moitra, and B. Sudakov. Nearly complete graphs decomposable into large induced matchings and their applications. In Proceedings of the 44th Symposium on Theory of Computing Conference, STOC 2012, New York, NY, USA, May 19 - 22, 2012, pages 1079-1090, 2012. 5

[5] S. Assadi. A two-pass (conditional) lower bound for semi-streaming maximum matching. In J. S. Naor and N. Buchbinder, editors, Proceedings of the 2022 ACM-SIAM Symposium on Discrete Algorithms, SODA 2022, Virtual Conference / Alexandria, VA, USA, January 9 12, 2022, pages 708-742. SIAM, 2022. 4

[6] S. Assadi, Y. Chen, and S. Khanna. Polynomial pass lower bounds for graph streaming algorithms. In Proceedings of the 51st Annual ACM SIGACT Symposium on Theory of Computing, STOC 2019, Phoenix, AZ, USA, June 23-26, 2019., pages 265-276, 2019. 1, 2, 3, 6, 7, 23

[7] S. Assadi, Y. Chen, and S. Khanna. Sublinear algorithms for $(\Delta+1)$ vertex coloring. In Proceedings of the Thirtieth Annual ACM-SIAM Symposium on Discrete Algorithms, SODA 2019, San Diego, California, USA, January 6-9, 2019, pages 767-786, 2019. 1 
[8] S. Assadi, S. Khanna, and Y. Li. Tight bounds for single-pass streaming complexity of the set cover problem. In Proceedings of the 48th Annual ACM SIGACT Symposium on Theory of Computing, STOC 2016, Cambridge, MA, USA, June 18-21, 2016, pages 698-711, 2016. 1

[9] S. Assadi, S. Khanna, and Y. Li. On estimating maximum matching size in graph streams. In Proceedings of the Twenty-Eighth Annual ACM-SIAM Symposium on Discrete Algorithms, SODA 2017, Barcelona, Spain, Hotel Porta Fira, January 16-19, pages 1723-1742, 2017. 3

[10] S. Assadi, S. Khanna, Y. Li, and G. Yaroslavtsev. Maximum matchings in dynamic graph streams and the simultaneous communication model. In Proceedings of the Twenty-Seventh Annual ACM-SIAM Symposium on Discrete Algorithms, SODA 2016, Arlington, VA, USA, January 10-12, 2016, pages 1345-1364, 2016. 3

[11] S. Assadi, G. Kol, R. R. Saxena, and H. Yu. Multi-pass graph streaming lower bounds for cycle counting, max-cut, matching size, and other problems. In S. Irani, editor, 61st IEEE Annual Symposium on Foundations of Computer Science, FOCS 2020, Durham, NC, USA, November 16-19, 2020, pages 354-364. IEEE, 2020. 4

[12] S. Assadi, G. Kol, and Z. Zhang. Rounds vs communication tradeoffs for maximal independent sets. In 63rd IEEE Annual Symposium on Foundations of Computer Science, FOCS 2022, Denver, CO, USA, October 31 - November 3, 2022, pages 1193-1204. IEEE, 2022. 4

[13] S. Assadi and V. N. Graph streaming lower bounds for parameter estimation and property testing via a streaming XOR lemma. In S. Khuller and V. V. Williams, editors, STOC '21: 53rd Annual ACM SIGACT Symposium on Theory of Computing, Virtual Event, Italy, June 21-25, 2021, pages 612-625. ACM, 2021. 4

[14] S. Assadi and R. Raz. Near-quadratic lower bounds for two-pass graph streaming algorithms. In S. Irani, editor, 61st IEEE Annual Symposium on Foundations of Computer Science, FOCS 2020, Durham, NC, USA, November 16-19, 2020, pages 342-353. IEEE, 2020. 4

[15] Z. Bar-Yossef, T. S. Jayram, R. Kumar, and D. Sivakumar. An information statistics approach to data stream and communication complexity. In 43rd Symposium on Foundations of Computer Science (FOCS 2002), 16-19 November 2002, Proceedings, pages 209-218, 2002. 5, 7

[16] B. Barak, M. Braverman, X. Chen, and A. Rao. How to compress interactive communication. In Proceedings of the 42nd ACM Symposium on Theory of Computing, STOC 2010, 5-8 June 2010, pages $67-76,2010.5$

[17] S. Baswana. Streaming algorithm for graph spanners - single pass and constant processing time per edge. Inf. Process. Lett., 106(3):110-114, 2008. 1, 3

[18] R. Becker, A. Karrenbauer, S. Krinninger, and C. Lenzen. Near-optimal approximate shortest paths and transshipment in distributed and streaming models. In 31st International Symposium on Distributed Computing, DISC 2017, October 16-20, 2017, Vienna, Austria, pages 7:1-7:16, 2017. 1, 3

[19] F. A. Behrend. On sets of integers which contain no three terms in arithmetical progression. Proceedings of the National Academy of Sciences of the United States of America, 32(12):331, 1946. 5 
[20] S. K. Bera and A. Chakrabarti. Towards tighter space bounds for counting triangles and other substructures in graph streams. In 34th Symposium on Theoretical Aspects of Computer Science, STACS 2017, March 8-11, 2017, Hannover, Germany, pages 11:1-11:14, 2017. 1

[21] S. K. Bera, A. Chakrabarti, and P. Ghosh. Graph coloring via degeneracy in streaming and other space-conscious models. In 47th International Colloquium on Automata, Languages, and Programming, ICALP 2020, July 8-11, 2020, Saarbrücken, Germany (Virtual Conference), pages 11:1-11:21, 2020. 1

[22] M. Braverman, F. Ellen, R. Oshman, T. Pitassi, and V. Vaikuntanathan. A tight bound for set disjointness in the message-passing model. In 54th Annual IEEE Symposium on Foundations of Computer Science, FOCS 2013, 26-29 October, 2013, Berkeley, CA, USA, pages 668-677, 2013. 5

[23] M. Braverman, A. Garg, D. Pankratov, and O. Weinstein. From information to exact communication. In Symposium on Theory of Computing Conference, STOC'13, June 1-4, 2013, pages $151-160,2013.7$

[24] M. Braverman and A. Moitra. An information complexity approach to extended formulations. In Symposium on Theory of Computing Conference, STOC'13, June 1-4, 2013, pages 161-170, 2013. 7

[25] M. Braverman and A. Rao. Information equals amortized communication. In IEEE 52nd Annual Symposium on Foundations of Computer Science, FOCS 2011, October 22-25, 2011, pages $748-757,2011.5$

[26] M. Braverman and O. Weinstein. An interactive information odometer and applications. In Proceedings of the Forty-Seventh Annual ACM on Symposium on Theory of Computing, STOC 2015, June 14-17, 2015, pages 341-350, 2015. 7

[27] L. Bulteau, V. Froese, K. Kutzkov, and R. Pagh. Triangle counting in dynamic graph streams. Algorithmica, 76(1):259-278, 2016. 1

[28] A. Chakrabarti, G. Cormode, and A. McGregor. Robust lower bounds for communication and stream computation. In Proceedings of the 40th Annual ACM Symposium on Theory of Computing, May 17-20, 2008, pages 641-650, 2008. 3

[29] A. Chakrabarti, P. Ghosh, A. McGregor, and S. Vorotnikova. Vertex ordering problems in directed graph streams. In Proceedings of the 2020 ACM-SIAM Symposium on Discrete Algorithms, SODA 2020, Salt Lake City, UT, USA, January 5-8, 2020, pages 1786-1802, 2020. 1, $2,3,4,25$

[30] A. Chakrabarti, Y. Shi, A. Wirth, and A. C. Yao. Informational complexity and the direct sum problem for simultaneous message complexity. In 42nd Annual Symposium on Foundations of Computer Science, FOCS 2001, 14-17 October 2001, pages 270-278, 2001. 5

[31] A. Chakrabarti and A. Wirth. Incidence geometries and the pass complexity of semi-streaming set cover. In Proceedings of the Twenty-Seventh Annual ACM-SIAM Symposium on Discrete Algorithms, SODA 2016, Arlington, VA, USA, January 10-12, 2016, pages 1365-1373, 2016. 1 
[32] Y. Chang, M. Farach-Colton, T. Hsu, and M. Tsai. Streaming complexity of spanning tree computation. In 37th International Symposium on Theoretical Aspects of Computer Science, STACS 2020, March 10-13, 2020, Montpellier, France, pages 34:1-34:19, 2020. 1, 3

[33] L. Chen, G. Kol, D. Paramonov, R. R. Saxena, Z. Song, and H. Yu. Almost optimal superconstant-pass streaming lower bounds for reachability. In S. Khuller and V. V. Williams, editors, STOC '21: 53rd Annual ACM SIGACT Symposium on Theory of Computing, Virtual Event, Italy, June 21-25, 2021, pages 570-583. ACM, 2021. 4

[34] L. Chen, G. Kol, D. Paramonov, R. R. Saxena, Z. Song, and H. Yu. Near-optimal twopass streaming algorithm for sampling random walks over directed graphs. In N. Bansal, E. Merelli, and J. Worrell, editors, 48th International Colloquium on Automata, Languages, and Programming, ICALP 2021, July 12-16, 2021, Glasgow, Scotland (Virtual Conference), volume 198 of LIPIcs, pages 52:1-52:19. Schloss Dagstuhl - Leibniz-Zentrum für Informatik, 2021. 4

[35] L. Chen, G. Kol, D. Paramonov, R. R. Saxena, Z. Song, and H. Yu. Towards multi-pass streaming lower bounds for optimal approximation of max-cut. In N. Bansal and V. Nagarajan, editors, Proceedings of the 2023 ACM-SIAM Symposium on Discrete Algorithms, SODA 2023, Florence, Italy, January 22-25, 2023, pages 878-924. SIAM, 2023. 4

[36] R. Chitnis, G. Cormode, H. Esfandiari, M. Hajiaghayi, A. McGregor, M. Monemizadeh, and S. Vorotnikova. Kernelization via sampling with applications to finding matchings and related problems in dynamic graph streams. In Proceedings of the Twenty-Seventh Annual ACM-SIAM Symposium on Discrete Algorithms, SODA 2016, January 10-12, 2016, pages 1326-1344, 2016. 2

[37] R. H. Chitnis, G. Cormode, M. T. Hajiaghayi, and M. Monemizadeh. Parameterized streaming: Maximal matching and vertex cover. In Proceedings of the Twenty-Sixth Annual ACM-SIAM Symposium on Discrete Algorithms, SODA 2015, San Diego, CA, USA, January 4-6, 2015, pages $1234-1251,2015.2$

[38] G. Cormode, J. Dark, and C. Konrad. Independent sets in vertex-arrival streams. In 46th International Colloquium on Automata, Languages, and Programming, ICALP 2019, July 912, 2019, Patras, Greece, pages 45:1-45:14, 2019. 1, 3

[39] G. Cormode and H. Jowhari. A second look at counting triangles in graph streams (corrected). Theor. Comput. Sci., 683:22-30, 2017. 1

[40] T. M. Cover and J. A. Thomas. Elements of information theory (2. ed.). Wiley, 2006. 33

[41] M. Crouch and D. S. Stubbs. Improved streaming algorithms for weighted matching, via unweighted matching. In Approximation, Randomization, and Combinatorial Optimization. Algorithms and Techniques, APPROX/RANDOM 2014, September 4-6, 2014, pages 96-104, 2014. 1

[42] D. P. Dubhashi and A. Panconesi. Concentration of Measure for the Analysis of Randomized Algorithms. Cambridge University Press, 2009. 4

[43] S. Eggert, L. Kliemann, and A. Srivastav. Bipartite graph matchings in the semi-streaming model. In Algorithms - ESA 2009, 17th Annual European Symposium, September 7-9, 2009. Proceedings, pages 492-503, 2009. 1 
[44] M. Elkin. Streaming and fully dynamic centralized algorithms for constructing and maintaining sparse spanners. ACM Trans. Algorithms, 7(2):20:1-20:17, 2011. 1, 3

[45] M. Elkin and J. Zhang. Efficient algorithms for constructing (1+, varepsilon; beta)-spanners in the distributed and streaming models. In Proceedings of the Twenty-Third Annual ACM Symposium on Principles of Distributed Computing, PODC 2004, St. John's, Newfoundland, Canada, July 25-28, 2004, pages 160-168, 2004. 1, 3

[46] Y. Emek and A. Rosén. Semi-streaming set cover - (extended abstract). In Automata, Languages, and Programming - 41st International Colloquium, ICALP 2014, Copenhagen, Denmark, July 8-11, 2014, Proceedings, Part I, pages 453-464, 2014. 1

[47] L. Epstein, A. Levin, J. Mestre, and D. Segev. Improved approximation guarantees for weighted matching in the semi-streaming model. SIAM J. Discrete Math., 25(3):1251-1265, 2011. 1

[48] J. Feigenbaum, S. Kannan, A. McGregor, S. Suri, and J. Zhang. On graph problems in a semi-streaming model. Theor. Comput. Sci., 348(2-3):207-216, 2005. 1, 2

[49] J. Feigenbaum, S. Kannan, A. McGregor, S. Suri, and J. Zhang. Graph distances in the data-stream model. SIAM J. Comput., 38(5):1709-1727, 2008. 1, 2, 3, 4

[50] E. Fischer, E. Lehman, I. Newman, S. Raskhodnikova, R. Rubinfeld, and A. Samorodnitsky. Monotonicity testing over general poset domains. In Proceedings on 34th Annual ACM Symposium on Theory of Computing, May 19-21, 2002, Montréal, Québec, Canada, pages 474-483, 2002. 5

[51] J. Fox, H. Huang, and B. Sudakov. On graphs decomposable into induced matchings of linear sizes. arXiv preprint arXiv:1512.07852, 2015. 5

[52] B. Gamlath, S. Kale, S. Mitrovic, and O. Svensson. Weighted matchings via unweighted augmentations. In Proceedings of the 2019 ACM Symposium on Principles of Distributed Computing, PODC 2019, Toronto, ON, Canada, July 29 - August 2, 2019, pages 491-500, 2019. 1

[53] M. Ghaffari, T. Gouleakis, C. Konrad, S. Mitrovic, and R. Rubinfeld. Improved massively parallel computation algorithms for mis, matching, and vertex cover. In Proceedings of the 2018 ACM Symposium on Principles of Distributed Computing, PODC 2018, July 23-27, 2018, pages 129-138, 2018. 1

[54] A. Goel, M. Kapralov, and S. Khanna. On the communication and streaming complexity of maximum bipartite matching. In Proceedings of the Twenty-third Annual ACM-SIAM Symposium on Discrete Algorithms, SODA '12, pages 468-485. SIAM, 2012. 1, 3, 5

[55] M. Göös, T. S. Jayram, T. Pitassi, and T. Watson. Randomized communication versus partition number. TOCT, 10(1):4:1-4:20, 2018. 7

[56] V. Guruswami and K. Onak. Superlinear lower bounds for multipass graph processing. In Proceedings of the 28th Conference on Computational Complexity, CCC 2013, K.lo Alto, California, USA, 5-7 June, 2013, pages 287-298, 2013. 1, 2, 3, 4

[57] B. V. Halldórsson, M. M. Halldórsson, E. Losievskaja, and M. Szegedy. Streaming algorithms for independent sets. In Automata, Languages and Programming, 37th International Colloquium, ICALP 2010, Bordeaux, France, July 6-10, 2010, Proceedings, Part I, pages 641-652, 2010. 1 
[58] S. Har-Peled, P. Indyk, S. Mahabadi, and A. Vakilian. Towards tight bounds for the streaming set cover problem. In Proceedings of the 35th ACM SIGMOD-SIGACT-SIGAI Symposium on Principles of Database Systems, PODS 2016, San Francisco, CA, USA, June 26 - July 01, 2016, pages 371-383, 2016. 1

[59] M. Henzinger, S. Krinninger, and D. Nanongkai. A deterministic almost-tight distributed algorithm for approximating single-source shortest paths. In Proceedings of the 48th Annual ACM SIGACT Symposium on Theory of Computing, STOC 2016, Cambridge, MA, USA, June 18-21, 2016, pages 489-498, 2016. 3

[60] M. R. Henzinger, P. Raghavan, and S. Rajagopalan. Computing on data streams. In External Memory Algorithms, Proceedings of a DIMACS Workshop, New Brunswick, New Jersey, USA, May 20-22, 1998, pages 107-118, 1998. 1, 2, 25

[61] T. S. Jayram, R. Kumar, and D. Sivakumar. Two applications of information complexity. In Proceedings of the 35th Annual ACM Symposium on Theory of Computing, June 9-11, 2003, San Diego, CA, USA, pages 673-682, 2003. 7

[62] S. Kale and S. Tirodkar. Maximum matching in two, three, and a few more passes over graph streams. In Approximation, Randomization, and Combinatorial Optimization. Algorithms and Techniques, APPROX/RANDOM 2017, August 16-18, 2017, Berkeley, CA, USA, pages 15:115:21, 2017. 1

[63] B. Kalyanasundaram and G. Schnitger. The probabilistic communication complexity of set intersection. SIAM J. Discrete Math., 5(4):545-557, 1992. 7

[64] M. Kapralov. Better bounds for matchings in the streaming model. In Proceedings of the Twenty-Fourth Annual ACM-SIAM Symposium on Discrete Algorithms, SODA 2013, New Orleans, Louisiana, USA, January 6-8, 2013, pages 1679-1697, 2013. 1, 3

[65] M. Kapralov, Y. T. Lee, C. Musco, C. Musco, and A. Sidford. Single pass spectral sparsification in dynamic streams. In 55th IEEE Annual Symposium on Foundations of Computer Science, FOCS 2014, Philadelphia, PA, USA, October 18-21, 2014, pages 561-570, 2014. 1

[66] M. Kapralov and D. P. Woodruff. Spanners and sparsifiers in dynamic streams. In ACM Symposium on Principles of Distributed Computing, PODC '14, Paris, France, July 15-18, 2014, pages 272-281, 2014. 1

[67] J. A. Kelner and A. Levin. Spectral sparsification in the semi-streaming setting. In 28th International Symposium on Theoretical Aspects of Computer Science, STACS 2011, March 10-12, 2011, Dortmund, Germany, pages 440-451, 2011. 1

[68] G. Kol, D. Paramonov, R. R. Saxena, and H. Yu. Characterizing the multi-pass streaming complexity for solving boolean csps exactly. In Y. T. Kalai, editor, 14 th Innovations in Theoretical Computer Science Conference, ITCS 2023, January 10-13, 2023, MIT, Cambridge, Massachusetts, USA, volume 251 of LIPIcs, pages 80:1-80:15. Schloss Dagstuhl - LeibnizZentrum für Informatik, 2023. 4

[69] C. Konrad. Maximum matching in turnstile streams. In Algorithms - ESA 2015 - 23rd Annual European Symposium, September 14-16, 2015, Proceedings, pages 840-852, 2015. 3 
[70] C. Konrad, F. Magniez, and C. Mathieu. Maximum matching in semi-streaming with few passes. In Approximation, Randomization, and Combinatorial Optimization. Algorithms and Techniques - 15th International Workshop, APPROX 2012, and 16th International Workshop, RANDOM 2012, Cambridge, MA, USA, August 15-17, 2012. Proceedings, pages 231-242, 2012. 1

[71] C. Konrad and K. K. Naidu. On two-pass streaming algorithms for maximum bipartite matching. In M. Wootters and L. Sanità, editors, Approximation, Randomization, and Combinatorial Optimization. Algorithms and Techniques, APPROX/RANDOM 2021, August 16-18, 2021, University of Washington, Seattle, Washington, USA (Virtual Conference), volume 207 of LIPIcs, pages 19:1-19:18. Schloss Dagstuhl - Leibniz-Zentrum für Informatik, 2021. 4

[72] E. Kushilevitz and N. Nisan. Communication complexity. Cambridge University Press, 1997. 5

[73] A. McGregor. Finding graph matchings in data streams. In Approximation, Randomization and Combinatorial Optimization, Algorithms and Techniques, 8th International Workshop on Approximation Algorithms for Combinatorial Optimization Problems, APPROX 2005 and 9th InternationalWorkshop on Randomization and Computation, RANDOM 2005, Berkeley, CA, USA, August 22-24, 2005, Proceedings, pages 170-181, 2005. 1

[74] A. McGregor. Graph stream algorithms: a survey. SIGMOD Record, 43(1):9-20, 2014. 1

[75] A. McGregor, S. Vorotnikova, and H. T. Vu. Better algorithms for counting triangles in data streams. In Proceedings of the 35th ACM SIGMOD-SIGACT-SIGAI Symposium on Principles of Database Systems, PODS 2016, San Francisco, CA, USA, June 26 - July 01, 2016, pages 401-411, 2016. 1

[76] S. Mukhopadhyay and D. Nanongkai. Weighted min-cut: sequential, cut-query, and streaming algorithms. In Proccedings of the 52nd Annual ACM SIGACT Symposium on Theory of Computing, STOC 2020, Chicago, IL, USA, June 22-26, 2020, pages 496-509, 2020. 1

[77] N. Nisan and A. Wigderson. Rounds in communication complexity revisited. In Proceedings of the 23rd Annual ACM Symposium on Theory of Computing, May 5-8, 1991, New Orleans, Louisiana, USA, pages 419-429, 1991. 3

[78] C. H. Papadimitriou and M. Sipser. Communication complexity. J. Comput. Syst. Sci., 28(2):260-269, 1984. 3

[79] A. Paz and G. Schwartzman. A $(2+\varepsilon)$-approximation for maximum weight matching in the semi-streaming model. In Proceedings of the Twenty-Eighth Annual ACM-SIAM Symposium on Discrete Algorithms, SODA 2017, Barcelona, Spain, Hotel Porta Fira, January 16-19, pages 2153-2161, 2017. 1

[80] S. Ponzio, J. Radhakrishnan, and S. Venkatesh. The communication complexity of pointer chasing: Applications of entropy and sampling. In Proceedings of the Thirty-First Annual ACM Symposium on Theory of Computing, May 1-4, 1999, Atlanta, Georgia, USA, pages 602-611, 1999. 3

[81] A. A. Razborov. On the distributional complexity of disjointness. Theor. Comput. Sci., 106(2):385-390, 1992. 7 
[82] A. Rubinstein, T. Schramm, and S. M. Weinberg. Computing exact minimum cuts without knowing the graph. In 9th Innovations in Theoretical Computer Science Conference, ITCS 2018, January 11-14, 2018, Cambridge, MA, USA, pages 39:1-39:16, 2018. 1, 2

[83] I. Z. Ruzsa and E. Szemerédi. Triple systems with no six points carrying three triangles. Combinatorics (Keszthely, 1976), Coll. Math. Soc. J. Bolyai, 18:939-945, 1978. 5

[84] A. D. Sarma, S. Gollapudi, and R. Panigrahy. Estimating pagerank on graph streams. J. ACM, 58(3):13:1-13:19, 2011. 1

[85] O. Weinstein and D. P. Woodruff. The simultaneous communication of disjointness with applications to data streams. In Automata, Languages, and Programming - 42nd International Colloquium, ICALP 2015, July 6-10, 2015, Proceedings, Part I, pages 1082-1093, 2015. 7

[86] A. C. Yao. Some complexity questions related to distributive computing (preliminary report). In Proceedings of the 11h Annual ACM Symposium on Theory of Computing, April 30 - May 2, 1979, Atlanta, Georgia, USA, pages 209-213, 1979. 5

[87] A. C. Yao. Lower bounds by probabilistic arguments (extended abstract). In 24th Annual Symposium on Foundations of Computer Science, Tucson, Arizona, USA, 7-9 November 1983, pages 420-428, 1983. 17

[88] A. Yehudayoff. Pointer chasing via triangular discrimination. Electronic Colloquium on Computational Complexity (ECCC), 23:151, 2016. 3

[89] M. Zelke. Intractability of min- and max-cut in streaming graphs. Inf. Process. Lett., 111(3):145-150, 2011. 1

\section{Appendix}

\section{A Basic Information Theory Facts}

Our proofs rely on basic concepts from information theory which we summarize below. We refer the interested reader to the excellent textbook by Cover and Thomas [40] for a broader introduction.

Fact A.1. Let A, B, C, and D be four (possibly correlated) random variables.

1. $0 \leqslant \mathbb{H}(\mathrm{A}) \leqslant \log |\operatorname{supp}(\mathrm{A})|$, where $\operatorname{supp}(\mathrm{A})$ denotes the support of $\mathrm{A}$.

2. $\mathbb{I}(\mathrm{A} ; \mathrm{B} \mid \mathrm{C}) \geqslant 0$. The equality holds iff $\mathrm{A} \perp \mathrm{B} \mid \mathrm{C}$.

3. $\mathbb{H}(\mathrm{A} \mid \mathrm{B}, \mathrm{C}) \leqslant \mathbb{H}(\mathrm{A} \mid \mathrm{B})$. The equality holds iff $\mathrm{A} \perp \mathrm{C} \mid \mathrm{B}$.

4. $\mathbb{I}(\mathrm{A}, \mathrm{B} ; \mathrm{C} \mid \mathrm{D})=\mathbb{I}(\mathrm{A} ; \mathrm{C} \mid \mathrm{D})+\mathbb{I}(\mathrm{B} ; \mathrm{C} \mid \mathrm{A}, \mathrm{D})$ (the chain rule of mutual information).

5. $\mathbb{I}(f(\mathrm{~A}) ; \mathrm{B} \mid \mathrm{C}) \leqslant \mathbb{I}(\mathrm{A} ; \mathrm{B} \mid \mathrm{C})$ for any deterministic function $f$ (the data processing inequality).

The above facts also immediately imply the following which we use in our proofs.

Fact A.2. $\mathbb{I}(A ; B \mid C) \leqslant \mathbb{H}(A \mid C) \leqslant \mathbb{H}(A) \leqslant \log |\operatorname{supp}(A)|$.

We will also use the following two standard inequalities regarding conditional mutual information. 
Proposition A.3. If $\mathrm{A} \perp \mathrm{D} \mid \mathrm{C}$, then, $\mathbb{I}(\mathrm{A} ; \mathrm{B} \mid \mathrm{C}) \leqslant \mathbb{I}(\mathrm{A} ; \mathrm{B} \mid \mathrm{C}, \mathrm{D})$.

Proof. By Fact A.1-(3), since A $\perp$ D $\mid$ C, we have $\mathbb{H}(A \mid C)=\mathbb{H}(A \mid C, D)$ and since conditioning can only decrease the entropy, $\mathbb{H}(A \mid C, B) \geqslant \mathbb{H}(A \mid C, B, D)$. As such,

$$
\mathbb{I}(\mathrm{A} ; \mathrm{B} \mid \mathrm{C})=\mathbb{H}(\mathrm{A} \mid \mathrm{C})-\mathbb{H}(\mathrm{A} \mid \mathrm{C}, \mathrm{B}) \leqslant \mathbb{H}(\mathrm{A} \mid \mathrm{C}, \mathrm{D})-\mathbb{H}(\mathrm{A} \mid \mathrm{C}, \mathrm{B}, \mathrm{D})=\mathbb{I}(\mathrm{A} ; \mathrm{B} \mid \mathrm{C}, \mathrm{D}) .
$$

Proposition A.4. If $\mathrm{A} \perp \mathrm{D} \mid \mathrm{B}, \mathrm{C}$, then, $\mathbb{I}(\mathrm{A} ; \mathrm{B} \mid \mathrm{C}) \geqslant \mathbb{I}(\mathrm{A} ; \mathrm{B} \mid \mathrm{C}, \mathrm{D})$.

Proof. By Fact A.1-(3), since A $\perp$ D $\mid B, C$, we have $\mathbb{H}(A \mid B, C)=\mathbb{H}(A \mid B, C, D)$ and since conditioning can only reduce the entropy, $\mathbb{H}(A \mid C) \geqslant \mathbb{H}(A \mid D, C)$. As such,

$$
\mathbb{I}(\mathrm{A} ; \mathrm{B} \mid \mathrm{C})=\mathbb{H}(\mathrm{A} \mid \mathrm{C})-\mathbb{H}(\mathrm{A} \mid \mathrm{B}, \mathrm{C}) \geqslant \mathbb{H}(\mathrm{A} \mid \mathrm{D}, \mathrm{C})-\mathbb{H}(\mathrm{A} \mid \mathrm{B}, \mathrm{C}, \mathrm{D})=\mathbb{I}(\mathrm{A} ; \mathrm{B} \mid \mathrm{C}, \mathrm{D}) \text {. }
$$

We shall also use the following measures of distance (or divergence) between distributions.

KL-divergence. For two distributions $\mu$ and $\nu$, the Kullback-Leibler divergence between $\mu$ and $\nu$ is denoted by $\mathbb{D}(\mu \| \nu)$ and defined as:

$$
\mathbb{D}(\mu \| \nu):=\underset{a \sim \mu}{\mathbb{E}}\left[\log \frac{\operatorname{Pr}_{\mu}(a)}{\operatorname{Pr}_{\nu}(a)}\right]
$$

We have the following relation between mutual information and KL-divergence.

Fact A.5. For random variables A, B, C,

$$
\mathbb{I}(\mathrm{A} ; \mathrm{B} \mid \mathrm{C})=\underset{\mathrm{B}, \mathrm{C}}{\mathbb{E}}[\mathbb{D}(\operatorname{dist}(\mathrm{A} \mid B, C) \| \operatorname{dist}(\mathrm{A} \mid C))] .
$$

Total variation distance. We denote the total variation distance between two distributions $\mu$ and $\nu$ on the same support $\Omega$ by $\|\mu-\nu\|_{\text {tvd }}$, defined as:

$$
\|\mu-\nu\|_{\mathrm{tvd}}:=\max _{\Omega^{\prime} \subseteq \Omega}\left(\mu\left(\Omega^{\prime}\right)-\nu\left(\Omega^{\prime}\right)\right)=\frac{1}{2} \cdot \sum_{x \in \Omega}|\mu(x)-\nu(x)| .
$$

Fact A.6. Suppose $\mu$ and $\nu$ are two distributions for a random variable A, then,

$$
\underset{\mu}{\mathbb{E}}[\mathrm{A}] \leqslant \underset{\nu}{\mathbb{E}}[\mathrm{A}]+\|\mu-\nu\|_{\mathrm{tvd}} \cdot \max |\mathrm{A}|
$$

The following Pinsker's inequality bounds the total variation distance between two distributions based on their KL-divergence,

Fact A.7 (Pinsker's inequality). For any distributions $\mu$ and $\nu,\|\mu-\nu\|_{\mathrm{tvd}} \leqslant \sqrt{\frac{1}{2} \cdot \mathbb{D}(\mu \| \nu)}$.

Finally, we use the following simple auxiliary result in our proofs.

Proposition A.8. Let $n$ be an even integer, $\mathcal{U}_{n}$ be the uniform distribution on $[n]$, and $\mu$ be any distribution on $[n]$ with $\left\|\mu-\mathcal{U}_{n}\right\|_{\mathrm{tvd}}=\delta$. Suppose $S$ is the top half of elements with the largest probability in $\mu$. Then, $\operatorname{Pr}_{\mu}(e \in S) \geqslant \frac{1}{2}+\frac{\delta}{2}$. 
Proof. Suppose without the loss of generality that $\mu(1) \geqslant \mu(2) \geqslant \cdots \geqslant \mu(n)$ by a renaming of elements. We thus have,

$$
\operatorname{Pr}_{\mu}(e \in S)=\sum_{i=1}^{n / 2} \mu(i)
$$

Let $\ell$ be the largest index such that $\mu(\ell) \geqslant \frac{1}{n}$. By the definition in Eq (15),

$$
\sum_{i=1}^{\ell}\left(\mu(i)-\frac{1}{n}\right)=\delta
$$

Case (a): when $\ell \leqslant n / 2$. We have,

$$
\begin{aligned}
\sum_{i=1}^{n / 2} \mu(i) & =\sum_{i=1}^{\ell} \mu(i)+\sum_{i=\ell+1}^{n / 2} \mu(i) \geqslant \sum_{i=1}^{\ell} \mu(i)+\frac{n / 2-\ell}{n-\ell} \cdot \sum_{i=\ell+1}^{n} \mu(i) \\
& =\sum_{i=1}^{\ell} \mu(i)+\frac{n / 2-\ell}{n-\ell} \cdot\left(1-\sum_{i=1}^{\ell} \mu(i)\right)=\sum_{i=1}^{\ell} \mu(i) \cdot\left(\frac{n / 2}{n-\ell}\right)+\frac{n / 2-\ell}{n-\ell} \\
& \geqslant\left(\frac{\ell}{n}+\delta\right) \cdot\left(\frac{n / 2}{n-\ell}\right)+\frac{n / 2-\ell}{n-\ell}=\frac{(n / 2) \cdot \ell+\left(n^{2} / 2\right) \cdot \delta+n^{2} / 2-n \cdot \ell}{n \cdot(n-\ell)} \quad \text { (by Eq (17)) } \\
& =\frac{n-\ell+n \cdot \delta}{2 \cdot(n-\ell)}=\frac{1}{2}+\frac{\delta \cdot n}{2 \cdot(n-\ell)} \geqslant \frac{1}{2}+\frac{\delta}{2},
\end{aligned}
$$

as $\ell \leqslant n / 2$. Plugging in this in Eq (16) proves the statement in this case.

Case (b): when $\ell>n / 2$. We have,

$$
\sum_{i=1}^{n / 2} \mu(i) \geqslant \frac{n / 2}{\ell} \cdot \sum_{i=1}^{\ell} \mu(i) \geqslant \frac{n / 2}{\ell} \cdot\left(\frac{\ell}{n}+\delta\right) \geqslant \frac{1}{2}+\frac{\delta}{2}, \quad(\text { as } \mu(1) \geqslant \cdots \geqslant \mu(\ell), \text { and by Eq (17)) }
$$

as $n / 2<\ell \leqslant n$. Plugging in this in Eq (16) proves this case and concludes the entire proof. 